Research Article

Nuttapol Pakkaranang*, Habib ur Rehman, and Wiyada Kumam

\title{
Two strongly convergent self-adaptive iterative schemes for solving pseudo-monotone equilibrium problems with applications
}

https://doi.org/10.1515/dema-2021-0030

received October 30, 2020; accepted July 6, 2021

Abstract: The aim of this paper is to propose two new modified extragradient methods to solve the pseudomonotone equilibrium problem in a real Hilbert space with the Lipschitz-type condition. The iterative schemes use a new step size rule that is updated on each iteration based on the value of previous iterations. By using mild conditions on a bi-function, two strong convergence theorems are established. The applications of proposed results are studied to solve variational inequalities and fixed point problems in the setting of real Hilbert spaces. Many numerical experiments have been provided in order to show the algorithmic performance of the proposed methods and compare them with the existing ones.

Keywords: equilibrium problem, pseudomonotone bifunction, Lipschitz-type conditions, strong convergence, variational inequality problems, fixed point problem

MSC 2020: 47J25, 47H09, 47H06, 47J05

\section{Introduction}

Assume that $\mathcal{K}$ is a convex subset of a real Hilbert space $\mathcal{E}$. Suppose that $f: \mathcal{E} \times \mathcal{E} \rightarrow \mathbb{R}$ satisfying $f\left(y_{1}, y_{1}\right)=0$ for each $y_{1} \in \mathcal{K}$ and the equilibrium problem (EP) [1,2] for $f$ on $\mathcal{K}$ is defined in the following manner:

$$
\text { Find } x^{*} \in \mathcal{K} \text { such that } f\left(x^{*}, y_{1}\right) \geq 0 \text {, for all } y_{1} \in \mathcal{K} \text {. }
$$

Let the solution of an EP be denoted by $E P(f, \mathcal{K})$ and $x^{*} \in E P(f, \mathcal{K})$. Let take $x^{*}=P_{E P(f, \mathcal{K})}(\theta)$, where $\theta$ stands for the zero element in $\mathcal{E}$. Next, we consider the different types of bi-function monotonicity (see $[1,3]$ for further information). A bi-function $f: \mathcal{E} \times \mathcal{E} \rightarrow \mathbb{R}$ on $\mathcal{K}$ for some $\xi>0$ is said to be

(1) strongly monotone if

$$
f\left(y_{1}, y_{2}\right)+f\left(y_{2}, y_{1}\right) \leq-\xi\left\|y_{1}-y_{2}\right\|^{2}, \quad \forall y_{1}, y_{2} \in \mathcal{K}
$$

\footnotetext{
* Corresponding author: Nuttapol Pakkaranang, Department of Mathematics, Faculty of Science and Technology, Phetchabun Rajabhat University, Phetchabun 67000, Thailand, e-mail: nuttapol.pak@pcru.ac.th

Habib ur Rehman: Department of Mathematics, King Mongkut's University of Technology Thonburi (KMUTT), Bangkok 10140, Thailand, e-mail: hrehman.hed@gmail.com

Wiyada Kumam: Program in Applied Statistics, Department of Mathematics and Computer Science, Faculty of Science and Technology, Rajamangala University of Technology Thanyaburi, Thanyaburi, Pathumthani 12110, Thailand,

e-mail: wiyada.kum@rmutt.ac.th
} 
(2) monotone if

$$
f\left(y_{1}, y_{2}\right)+f\left(y_{2}, y_{1}\right) \leq 0, \quad \forall y_{1}, y_{2} \in \mathcal{K} ;
$$

(3) strongly pseudo-monotone if

$$
f\left(y_{1}, y_{2}\right) \geq 0 \Rightarrow f\left(y_{2}, y_{1}\right) \leq-\xi\left\|y_{1}-y_{2}\right\|^{2}, \quad \forall y_{1}, y_{2} \in \mathcal{K} ;
$$

(4) pseudo-monotone if

$$
f\left(y_{1}, y_{2}\right) \geq 0 \Rightarrow f\left(y_{2}, y_{1}\right) \leq 0, \quad \forall y_{1}, y_{2} \in \mathcal{K}
$$

Let $f: \mathcal{E} \times \mathcal{E} \rightarrow \mathbb{R}$ be satisfying the Lipschitz-type condition [4] on $\mathcal{K}$ if there exist two constants $c_{1}, c_{2}>0$, such that

$$
f\left(y_{1}, y_{3}\right) \leq f\left(y_{1}, y_{2}\right)+f\left(y_{2}, y_{3}\right)+c_{1}\left\|y_{1}-y_{2}\right\|^{2}+c_{2}\left\|y_{2}-y_{3}\right\|^{2}, \quad \forall y_{1}, y_{2}, y_{3} \in \mathcal{K} .
$$

The unique format of (EP) unifies a number of mathematical problems such as fixed-point problem, variational inequality problems, vector and scalar minimization problems, the complementarity problems, the saddle points problems, the Nash EP in non-cooperative games and the inverse optimization problems $[1,5,6]$. The problem (EP) is also taken as the Ky Fan inequality due to previous contributions [2]. Many iterative methods have been proposed and studied to solve different classes of (EP). Many effective iterative methods have been already established along with their convergence analysis [7-13] and others in [14-23].

The regularization method is the most effective technique to solve many ill-possessed problems in different fields of pure and applied mathematics. The key advantage of the regularization method is that it can solve monotone EPs and transform the original problem into a strongly monotone equilibrium subproblem. Therefore, each sub-problem is strongly monotone and there is a unique solution. In particular, the sub-problem can be resolved more easily than the original problem, and the regularization solutions converge to some solution of the initial problem once the regularization variables appear to have an appropriate limit. The two common regularization methods are proximal point method and Tikhonov's regularized method. These two methods have recently been used to solve the (EP) [24-27].

The proximal method [28] is an effective method to solve EPs and need to solve minimization problems on each iterative step. This method was also known as the two-step extragradient method in [29] due to the previous contribution of Korpelevich extragradient method [30] to solve the saddle point problems. Tran et al. in [29] introduced a sequence of $\left\{x_{n}\right\}$ as follows:

$$
\left\{\begin{array}{l}
x_{0} \in \mathcal{K}, \\
y_{n}=\underset{y \in \mathcal{K}}{\arg \min }\left\{\zeta f\left(x_{n}, y\right)+\frac{1}{2}\left\|x_{n}-y\right\|^{2}\right\}, \\
x_{n+1}=\underset{y \in \mathcal{K}}{\arg \min }\left\{\zeta f\left(y_{n}, y\right)+\frac{1}{2}\left\|x_{n}-y\right\|^{2}\right\},
\end{array}\right.
$$

where $0<\zeta<\min \left\{\frac{1}{2 c_{1}}, \frac{1}{2 c_{2}}\right\}$.

Recently, Wang et al. in [31] introduced a non-convex combination iterative method to solve pseudomonotone EPs. Strong convergence of iterative sequences is the main contribution of the proposed method. The detailed method is as follows: choose $0<\zeta_{n}<\min \left\{\frac{1}{2 c_{1}}, \frac{1}{2 c_{2}}\right\}, \delta_{n} \subset[\delta, 1)$ with $0<\delta<1$ and $\alpha_{n}$ such that

$$
\lim _{n \rightarrow \infty} \alpha_{n}=0 \text { and } \sum_{n=1}^{\infty} \alpha_{n}=\infty \text {. }
$$

Compute $x_{n+1}=P_{\mathcal{K}}\left[\alpha_{n} x_{n}+\left(1-\alpha_{n}\right) z_{n}-\alpha_{n} \delta_{n} x_{n}\right]$, where

$$
\left\{\begin{array}{l}
y_{n}=\underset{y \in \mathcal{K}}{\arg \min }\left\{\zeta_{n} f\left(x_{n}, y\right)+\frac{1}{2}\left\|x_{n}-y\right\|^{2}\right\}, \\
z_{n}=\underset{y \in \mathcal{K}}{\arg \min }\left\{\zeta_{n} f\left(y_{n}, y\right)+\frac{1}{2}\left\|x_{n}-y\right\|^{2}\right\} .
\end{array}\right.
$$


A natural question arises.

Is it possible to introduce a modified version of method (1.1) in the sense that constant step size took over with the nonmonotonic step size rule and it improve the numerical efficiency of the method"?

In this paper, we provide a positive answer to the above question, that is, the gradient method provides a strong convergence sequence by using a non-monotonic step size rule for solving EPs accompanied with pseudo-monotone bi-functions. Motivated by the works of Censor et al. [32] and Wang et al. [31] we introduce a new gradient-type method to figure out the problem (EP) in the setting of an infinite-dimensional real Hilbert space. Some applications on the topic of the variational inequality problem and the fixed point problem are provided. Numerical experiments have described that the proposed methods are more successful than the given one in [31].

The rest of this article has been arranged as follows: Section 2 contains some basic definitions and identities used in this paper. Sections 3 and 4 include the proposed methods as well as the convergence theorems. Section 5 presents an application of our results to solve variational inequalities and the fixed point problems. Section 6 sets out numerical explanations which demonstrate the computational effectiveness of the proposed methods.

\section{Preliminaries}

For a convex function $h: \mathcal{K} \rightarrow \mathbb{R}$ subdifferential of $h$ at $y_{1} \in \mathcal{K}$ is defined by

$$
\partial h\left(y_{1}\right)=\left\{y_{3} \in \mathcal{E}: h\left(y_{2}\right)-h\left(y_{1}\right) \geq\left\langle y_{3}, y_{2}-y_{1}\right\rangle, \quad \forall y_{2} \in \mathcal{K}\right\} .
$$

A normal cone of $\mathcal{K}$ at $y_{1} \in \mathcal{K}$ is defined by

$$
N_{\mathcal{K}}\left(y_{1}\right)=\left\{y_{3} \in \mathcal{E}:\left\langle y_{3}, y_{2}-y_{1}\right\rangle \leq 0, \quad \forall y_{2} \in \mathcal{K}\right\} .
$$

Lemma 2.1. [33] Assume that $h: \mathcal{K} \rightarrow \mathbb{R}$ is lower semi-continuous, convex and subdifferentiable function on $\mathcal{K}$. Then, $y_{1} \in \mathcal{K}$ is a minimizer of a function $h$ if and only if

$$
0 \in \partial h\left(y_{1}\right)+N_{\mathcal{K}}\left(y_{1}\right),
$$

where $\partial h\left(y_{1}\right)$ and $N_{\mathcal{K}}\left(y_{1}\right)$ denote the subdifferential of $h$ at $y_{1} \in \mathcal{K}$ and the normal cone of $\mathcal{K}$ at $y_{1}$, respectively.

Definition 2.2. [34] The metric projection $P_{\mathcal{K}}\left(y_{1}\right)$ for $y_{1} \in \mathcal{E}$ onto a closed and convex subset $\mathcal{K}$ of $\mathcal{E}$ is defined by

$$
P_{\mathcal{K}}\left(y_{1}\right)=\arg \min \left\{\left\|y_{2}-y_{1}\right\|: y_{2} \in \mathcal{K}\right\} .
$$

Lemma 2.3. [35] Let $P_{\mathcal{K}}: \mathcal{E} \rightarrow \mathcal{K}$ be a metric projection on $\mathcal{K}$. Then

(1) For each $y_{2} \in \mathcal{K}$ and $y_{1} \in \mathcal{E}$ such that

$$
\left\|y_{1}-P_{\mathcal{K}}\left(y_{1}\right)\right\| \leq\left\|y_{1}-y_{2}\right\|^{2}
$$

(2) $y_{3}=P_{\mathcal{K}}\left(y_{1}\right)$ if and only if

$$
\left\langle y_{1}-y_{3}, y_{2}-y_{3}\right\rangle \leq 0, \quad \forall y_{2} \in \mathcal{K}
$$

Lemma 2.4. [36] For each $y_{1}, y_{2} \in \mathcal{E}$ with $\chi \in \mathbb{R}$, we have

$$
\left\|\chi y_{1}+(1-\chi) y_{2}\right\|^{2}=\chi\left\|y_{1}\right\|^{2}+(1-\chi)\left\|y_{2}\right\|^{2}-\chi(1-\chi)\left\|y_{1}-y_{2}\right\|^{2}
$$

and

$$
\left\|y_{1}+y_{2}\right\|^{2} \leq\left\|y_{1}\right\|^{2}+2\left\langle y_{2}, y_{1}+y_{2}\right\rangle \text {. }
$$


Lemma 2.5. [37] Let a sequence $\left\{\chi_{n}\right\}$ of non-negative real numbers such that

$$
\chi_{n+1} \leq\left(1-\tau_{n}\right) \chi_{n}+\tau_{n} \delta_{n}, \quad \forall n \in \mathbb{N},
$$

where $\left\{\tau_{n}\right\} \subset(0,1)$ and $\left\{\delta_{n}\right\} \subset \mathbb{R}$ satisfy the following conditions:

$$
\lim _{n \rightarrow \infty} \tau_{n}=0, \quad \sum_{n=1}^{\infty} \tau_{n}=\infty, \quad \text { and } \quad \limsup _{n \rightarrow \infty} \delta_{n} \leq 0 .
$$

Then, $\lim _{n \rightarrow \infty} \chi_{n}=0$.

Lemma 2.6. [38] Let $\left\{\chi_{n}\right\}$ be a sequence of real numbers such that there exists a subsequence $\left\{n_{i}\right\}$ of $\{n\}$ such that $\chi_{n_{i}}<\chi_{n_{i+1}}$ for all $i \in \mathbb{N}$. Then, there exists a non-decreasing sequence $m_{k} \subset \mathbb{N}$ such that $m_{k} \rightarrow \infty$ as $k \rightarrow \infty$, and the following conditions are fulfilled by all (sufficiently large) numbers $k \in \mathbb{N}$ :

$$
\chi_{m_{k}} \leq \chi_{m_{k+1}} \text { and } \chi_{k} \leq \chi_{m_{k+1}} \text {. }
$$

In fact, $m_{k}=\max \left\{j \leq k: \chi_{j} \leq \chi_{j+1}\right\}$.

Let $f: \mathcal{E} \times \mathcal{E} \rightarrow \mathbb{R}$ satisfy the following conditions:

(C1) $f\left(y_{2}, y_{2}\right)=0$ for all $y_{2} \in \mathcal{K}$ and $f$ is pseudo-monotone on $\mathcal{K}$;

(C2) $f$ satisfies the Lipschitz-type conditions on $\mathcal{E}$ with constants $c_{1}>0$ and $c_{2}>0$;

(C3) $f\left(y_{1}, y_{2}\right)$ is jointly weakly continuous on $\mathcal{E} \times \mathcal{E}$;

(C4) $f\left(y_{1}\right.$, .) is subdifferentiable and convex over $\mathcal{E}$ for each $x \in \mathcal{E}$.

\section{Explicit subgradient extragradient method and its convergence analysis}

The following is the first method in detail.

\section{Algorithm 1}

Initialization: Let $x_{1} \in \mathcal{K}, \sigma<\min \left\{1, \frac{1}{2 c_{1}}, \frac{1}{2 c_{2}}\right\}, \mu \in(0, \sigma), \zeta_{1}>0, \delta_{n} \subset[\delta, 1)$ with $0<\delta<1$ and $\alpha_{n} \subset(0,1)$ such that

$$
\lim _{n \rightarrow \infty} \alpha_{n}=0 \text { and } \quad \sum_{n=1}^{\infty} \alpha_{n}=\infty
$$

Step 1: Compute

$$
y_{n}=\underset{y \in \mathcal{K}}{\arg \min }\left\{\zeta_{n} f\left(x_{n}, y\right)+\frac{1}{2}\left\|x_{n}-y\right\|^{2}\right\} .
$$

If $x_{n}=y_{n}$, then STOP. Otherwise, go to the next step.

Step 2: Construct a half-space

$$
\mathcal{E}_{n}=\left\{z \in \mathcal{E}:\left\langle x_{n}-\zeta_{n} \omega_{n}-y_{n}, z-y_{n}\right\rangle \leq 0\right\},
$$

where $\omega_{n} \in \partial f\left(x_{n}, y_{n}\right)$ and compute

$$
z_{n}=\underset{y \in \mathcal{E}_{n}}{\arg \min }\left\{\mu \zeta_{n} f\left(y_{n}, y\right)+\frac{1}{2}\left\|x_{n}-y\right\|^{2}\right\} .
$$


Step 3: Compute

$$
x_{n+1}=P_{\mathcal{K}}\left[\alpha_{n} x_{n}+\left(1-\alpha_{n}\right) z_{n}-\alpha_{n} \delta_{n} x_{n}\right]
$$

Step 4: Revise the step size rule as follows:

$$
\zeta_{n+1}=\left\{\begin{array}{c}
\min \left\{\sigma, \frac{\mu f\left(y_{n}, z_{n}\right)}{f\left(x_{n}, z_{n}\right)-f\left(x_{n}, y_{n}\right)-c_{1}\left\|x_{n}-y_{n}\right\|^{2}-c_{2}\left\|z_{n}-y_{n}\right\|^{2}+1}\right\}, \\
\text { if } \frac{\mu f\left(y_{n}, z_{n}\right)}{f\left(x_{n}, z_{n}\right)-f\left(x_{n}, y_{n}\right)-c_{1}\left\|x_{n}-y_{n}\right\|^{2}-c_{2}\left\|z_{n}-y_{n}\right\|^{2}+1}>0, \\
\sigma \quad \text { else. }
\end{array}\right.
$$

Put $n:=n+1$ and go back to Step 1 .

Remark 3.1. The iteration $\zeta_{n+1}$ in (3.1) is well-defined and

$$
\zeta_{n+1}\left(f\left(x_{n}, z_{n}\right)-f\left(x_{n}, y_{n}\right)-c_{1}\left\|x_{n}-y_{n}\right\|^{2}-c_{2}\left\|y_{n}-z_{n}\right\|^{2}\right) \leq \mu f\left(y_{n}, z_{n}\right) .
$$

Lemma 3.2. Let $f: \mathcal{E} \times \mathcal{E} \rightarrow \mathbb{R}$ be a bi-function satisfying conditions (C1)-(C4). Then, for any $x^{*} \in E P(f, \mathcal{K}) \neq \varnothing$, we have

$$
\left\|z_{n}-x^{*}\right\|^{2} \leq\left\|x_{n}-x^{*}\right\|^{2}-\left(1-\zeta_{n+1}\right)\left\|z_{n}-x_{n}\right\|^{2}-\zeta_{n+1}\left(1-2 c_{1} \zeta_{n}\right)\left\|x_{n}-y_{n}\right\|^{2}-\zeta_{n+1}\left(1-2 c_{2} \zeta_{n}\right)\left\|z_{n}-y_{n}\right\|^{2} .
$$

Proof. The value of $z_{n}$ and Lemma 2.1 give that

$$
0 \in \partial_{2}\left\{\mu \zeta_{n} f\left(y_{n}, y\right)+\frac{1}{2}\left\|x_{n}-y\right\|^{2}\right\}\left(z_{n}\right)+N_{\mathcal{E}_{n}}\left(z_{n}\right)
$$

From above $\omega_{n} \in \partial_{2} f\left(y_{n}, z_{n}\right)$ and $\bar{\omega}_{n} \in N_{\mathcal{E}_{n}}\left(z_{n}\right)$, we have

$$
\mu \zeta_{n} \omega_{n}+z_{n}-x_{n}+\bar{\omega}_{n}=0 .
$$

Thus, we have since $\bar{\omega}_{n} \in N_{\mathcal{E}_{n}}\left(z_{n}\right),\left\langle\bar{\omega}_{n}, y-z_{n}\right\rangle \leq 0, \forall y \in \mathcal{E}_{n}$. It implies that

$$
\mu \zeta_{n}\left\langle\omega_{n}, y-z_{n}\right\rangle \geq\left\langle x_{n}-z_{n}, y-z_{n}\right\rangle, \quad \forall y \in \mathcal{E}_{n} .
$$

By $\omega_{n} \in \partial_{2} f\left(y_{n}, z_{n}\right)$, we obtain

$$
f\left(y_{n}, y\right)-f\left(y_{n}, z_{n}\right) \geq\left\langle\omega_{n}, y-z_{n}\right\rangle, \quad \forall y \in \mathcal{E} .
$$

Combining expressions (3.3) and (3.4) gives that

$$
\mu \zeta_{n} f\left(y_{n}, y\right)-\mu \zeta_{n} f\left(y_{n}, z_{n}\right) \geq\left\langle x_{n}-z_{n}, y-z_{n}\right\rangle, \quad \forall y \in \mathcal{E}_{n} .
$$

Substitution by $y=x^{*}$ in (3.5), we get

$$
\mu \zeta_{n} f\left(y_{n}, x^{*}\right)-\mu \zeta_{n} f\left(y_{n}, z_{n}\right) \geq\left\langle x_{n}-z_{n}, x^{*}-z_{n}\right\rangle, \quad \forall y \in \mathcal{E}_{n} .
$$

Since $x^{*} \in E P(f, \mathcal{K}), f\left(x^{*}, y_{n}\right) \geq 0$ and because of condition (C1) we have $f\left(y_{n}, x^{*}\right) \leq 0$. It implies that

$$
\left\langle x_{n}-z_{n}, z_{n}-x^{*}\right\rangle \geq \mu \zeta_{n} f\left(y_{n}, z_{n}\right) .
$$

Combining expressions (3.2) and (3.7), we have

$$
\left\langle x_{n}-z_{n}, z_{n}-x^{*}\right\rangle \geq \zeta_{n+1}\left[\zeta_{n}\left\{f\left(x_{n}, z_{n}\right)-f\left(x_{n}, y_{n}\right)\right\}-c_{1} \zeta_{n}\left\|x_{n}-y_{n}\right\|^{2}-c_{2} \zeta_{n}\left\|z_{n}-y_{n}\right\|^{2}\right] .
$$


Due to $z_{n} \in \mathcal{E}_{n}$, we have

$$
\zeta_{n}\left\langle\omega_{n}, z_{n}-y_{n}\right\rangle \geq\left\langle x_{n}-y_{n}, z_{n}-y_{n}\right\rangle
$$

From $\omega_{n} \in \partial_{2} f\left(x_{n}, y_{n}\right)$ and $y=z_{n}$, we have

$$
f\left(x_{n}, z_{n}\right)-f\left(x_{n}, y_{n}\right) \geq\left\langle\omega_{n}, z_{n}-y_{n}\right\rangle, \quad \forall y \in \mathcal{E} .
$$

Combining expressions (3.9) and (3.10), we obtain

$$
\zeta_{n}\left\{f\left(x_{n}, z_{n}\right)-f\left(x_{n}, y_{n}\right)\right\} \geq\left\langle x_{n}-y_{n}, z_{n}-y_{n}\right\rangle .
$$

Expressions (3.8) and (3.11) imply that

$$
2\left\langle x_{n}-z_{n}, z_{n}-x^{*}\right\rangle \geq \zeta_{n+1}\left[2\left\langle x_{n}-y_{n}, z_{n}-y_{n}\right\rangle-2 c_{1} \zeta_{n}\left\|x_{n}-y_{n}\right\|^{2}-2 c_{2} \zeta_{n}\left\|z_{n}-y_{n}\right\|^{2}\right] .
$$

We have the following facts:

$$
\begin{aligned}
2\left\langle x_{n}-z_{n}, z_{n}-x^{*}\right\rangle & =\left\|x_{n}-x^{*}\right\|^{2}-\left\|z_{n}-x_{n}\right\|^{2}-\left\|z_{n}-x^{*}\right\|^{2} . \\
2\left\langle x_{n}-y_{n}, z_{n}-y_{n}\right\rangle & =\left\|x_{n}-y_{n}\right\|^{2}+\left\|z_{n}-y_{n}\right\|^{2}-\left\|x_{n}-z_{n}\right\|^{2} .
\end{aligned}
$$

Combining above inequalities with (3.12) completes the proof.

Theorem 3.3. Let $\left\{x_{n}\right\}$ be a sequence generated by Algorithm 1 and the solution set EP( $f$, K) is non-empty. Then, the sequence $\left\{x_{n}\right\}$ is strongly convergent to an element $x^{*} \in \operatorname{EP}(f, \mathcal{K})$.

Proof. From Lemma 3.2, we have

$$
\left\|z_{n}-x^{*}\right\|^{2} \leq\left\|x_{n}-x^{*}\right\|^{2}, \quad \forall n \geq 2
$$

Next, we prove that $\left\{x_{n}\right\}$ is bounded. For all $n \geq 2$, with Lemma 2.4 (i), we obtain

$$
\begin{aligned}
\left\|x_{n+1}-x^{*}\right\|^{2}= & \left\|P_{\mathcal{K}}\left[\alpha_{n}\left(1-\delta_{n}\right) x_{n}+\left(1-\alpha_{n}\right) z_{n}\right]-P_{\mathcal{K}}\left(x^{*}\right)\right\|^{2} \\
\leq & \left\|\alpha_{n}\left(1-\delta_{n}\right) x_{n}+\left(1-\alpha_{n}\right) z_{n}-x^{*}\right\|^{2} \\
= & \left\|\alpha_{n}\left[\left(1-\delta_{n}\right) x_{n}-x^{*}\right]+\left(1-\alpha_{n}\right)\left(z_{n}-x^{*}\right)\right\|^{2} \\
\leq & \alpha_{n}\left\|\left(1-\delta_{n}\right) x_{n}-x^{*}\right\|^{2}+\left(1-\alpha_{n}\right)\left\|z_{n}-x^{*}\right\|^{2} \\
\leq & \alpha_{n}\left[\left\|\left(1-\delta_{n}\right)\left(x_{n}-x^{*}\right)+\delta_{n} x^{*}\right\|^{2}\right]+\left(1-\alpha_{n}\right)\left\|x_{n}-x^{*}\right\|^{2} \\
& -\left(1-\alpha_{n}\right)\left[\left(1-\zeta_{n+1}\right)\left\|z_{n}-x_{n}\right\|^{2}+\zeta_{n+1}\left(1-2 c_{1} \zeta_{n}\right)\left\|x_{n}-y_{n}\right\|^{2}+\zeta_{n+1}\left(1-2 c_{2} \zeta_{n}\right)\left\|z_{n}-y_{n}\right\|^{2}\right] \\
\leq & \alpha_{n}\left[\left(1-\delta_{n}\right)\left\|x_{n}-x^{*}\right\|^{2}+\delta_{n}\left\|x^{*}\right\|^{2}\right]+\left(1-\alpha_{n}\right)\left\|x_{n}-x^{*}\right\|^{2} \\
& -\left(1-\alpha_{n}\right)\left[(1-\sigma)\left\|z_{n}-x_{n}\right\|^{2}+\sigma\left(1-2 c_{1} \sigma\right)\left\|x_{n}-y_{n}\right\|^{2}+\sigma\left(1-2 c_{2} \sigma\right)\left\|z_{n}-y_{n}\right\|^{2}\right] \\
= & \left(1-\alpha_{n} \delta_{n}\right)\left\|x_{n}-x^{*}\right\|^{2}+\alpha_{n} \delta_{n}\left\|x^{*}\right\|^{2} \\
\leq & \max \left\{\left\|x_{n}-x^{*}\right\|^{2},\left\|x^{*}\right\|^{2}\right\} \leq \max \left\{\left\|x_{2}-x^{*}\right\|^{2},\left\|x^{*}\right\|^{2}\right\} .
\end{aligned}
$$

Thus, $\left\{x_{n}\right\}$ is a bounded sequence as well as $\left\{y_{n}\right\},\left\{z_{n}\right\}$ bounded. Let $q_{n}=\alpha_{n} x_{n}+\left(1-\alpha_{n}\right) z_{n}$, for every $n \in \mathbb{N}$. By Lemma 2.4(i), we have

$$
\left\|q_{n}-x^{*}\right\|^{2}=\left\|\alpha_{n} x_{n}+\left(1-\alpha_{n}\right) z_{n}-x^{*}\right\|^{2} \leq\left\|x_{n}-x^{*}\right\|^{2}, \quad \forall n \geq 2 .
$$

Thus, we obtain

$$
x_{n+1}=P_{\mathcal{K}}\left(q_{n}-\alpha_{n} \delta_{n} x_{n}\right)=P_{\mathcal{K}}\left[\left(1-\alpha_{n} \delta_{n}\right) q_{n}+\alpha_{n} \delta_{n}\left(1-\alpha_{n}\right)\left(z_{n}-x_{n}\right)\right] .
$$

By Lemma 2.4(ii) and (3.16), (3.17), we have (see equation (3.6) [31])

$$
\begin{aligned}
\left\|x_{n+1}-x^{*}\right\|^{2}= & \left\|P_{\mathcal{K}}\left[\left(1-\alpha_{n} \delta_{n}\right) q_{n}+\alpha_{n} \delta_{n}\left(1-\alpha_{n}\right)\left(z_{n}-x_{n}\right)\right]-P_{\mathcal{K}}\left(x^{*}\right)\right\|^{2} \\
\leq & \left(1-\alpha_{n} \delta_{n}\right)\left\|x_{n}-x^{*}\right\|^{2}+2 \alpha_{n} \delta_{n}\left(1-\alpha_{n}\right)\left\langle z_{n}-x_{n},\left(1-\alpha_{n} \delta_{n}\right) q_{n}+\alpha_{n} \delta_{n}\left(1-\alpha_{n}\right)\left(z_{n}-x_{n}\right)-x^{*}\right\rangle \\
& +2 \alpha_{n} \delta_{n}\left(1-\alpha_{n}\right)\left\langle-x^{*}, z_{n}-x_{n}\right\rangle+2 \alpha_{n} \delta_{n}\left\langle-x^{*}, x_{n}-x^{*}\right\rangle+2 \alpha_{n}^{2} \delta_{n}^{2}\left\langle x^{*}, x_{n}\right\rangle .
\end{aligned}
$$


The rest of the proof will be divided into the following two parts:

Case 1. Assume there is $n_{2} \in \mathbb{N}\left(n_{2} \geq 2\right)$ such that

$$
\left\|x_{n+1}-x^{*}\right\| \leq\left\|x_{n}-x^{*}\right\|, \quad \forall n \geq n_{2} .
$$

This implies that $\lim _{n \rightarrow \infty}\left\|x_{n}-x^{*}\right\|$ exists and let $\lim _{n \rightarrow \infty}\left\|x_{n}-x^{*}\right\|=l$. Thus, expression (3.14) $\left(\forall n \geq n_{2}\right)$ implies that

$$
\begin{aligned}
& (1-\sigma)\left\|z_{n}-x_{n}\right\|^{2}+\sigma\left(1-2 c_{1} \sigma\right)\left\|x_{n}-y_{n}\right\|^{2}+\sigma\left(1-2 c_{2} \sigma\right)\left\|z_{n}-y_{n}\right\|^{2} \\
& \quad \leq\left\|x_{n}-x^{*}\right\|^{2}-\left\|x_{n+1}-x^{*}\right\|^{2}+\alpha_{n}\left\|x^{*}\right\|^{2}+\alpha_{n} A_{0},
\end{aligned}
$$

where $A_{0}$ is the finite number

$$
A_{0}=\sup \left\{\left[(1-\sigma)\left\|z_{n}-x_{n}\right\|^{2}+\sigma\left(1-2 c_{1} \sigma\right)\left\|x_{n}-y_{n}\right\|^{2}+\sigma\left(1-2 c_{2} \sigma\right)\left\|z_{n}-y_{n}\right\|^{2}\right]: \forall n \in \mathbb{N}\right\} .
$$

The existence of $\lim _{n \rightarrow \infty}\left\|x_{n}-x^{*}\right\|=l$, with expression (3.19) implies that

$$
\lim _{n \rightarrow \infty}\left\|x_{n}-z_{n}\right\|=\lim _{n \rightarrow \infty}\left\|x_{n}-y_{n}\right\|=\lim _{n \rightarrow \infty}\left\|z_{n}-y_{n}\right\|=0 .
$$

Since the sequences $\left\{x_{n}\right\},\left\{y_{n}\right\}$ and $\left\{z_{n}\right\}$ are bounded, there exists a subsequence $\left\{x_{n_{k}}\right\}$ of $\left\{x_{n}\right\}$ such that $\left\{x_{n_{k}}\right\}$ converges weakly to some $x \in \mathcal{K}$ and

$$
\limsup _{n \rightarrow \infty}\left\langle-x^{*}, x_{n}-x^{*}\right\rangle=\limsup _{k \rightarrow \infty}\left\langle-\chi^{*}, x_{n_{k}}-x^{*}\right\rangle=\left\langle-\chi^{*}, x-x^{*}\right\rangle .
$$

By expression (3.20), the subsequences $\left\{y_{n_{k}}\right\}$ and $\left\{z_{n_{k}}\right\}$ weakly converge to $x$ as $k \rightarrow \infty$. From (3.5), we have

$$
\mu \zeta_{n_{k}} f\left(y_{n_{k}}, y\right)-\mu \zeta_{n_{k}} f\left(y_{n_{k}}, z_{n_{k}}\right) \geq\left\langle x_{n_{k}}-z_{n_{k}}, y-z_{n_{k}}\right\rangle, \quad \forall y \in \mathcal{E}_{n}
$$

By letting $k \rightarrow \infty$, it implies that

$$
f(x, y) \geq 0, \quad \forall y \in \mathcal{K} \subset \mathcal{E}_{n} .
$$

It follows that $x \in E P(f, \mathcal{K})$. In conclusion, by (3.21) and Lemma 2.3(ii), we get

$$
\begin{aligned}
\limsup _{n \rightarrow \infty}\left\langle-\chi^{*}, x_{n}-\chi^{*}\right\rangle & =\underset{k \rightarrow \infty}{\lim \sup }\left\langle-\chi^{*}, x_{n_{k}}-\chi^{*}\right\rangle=\left\langle-\chi^{*}, x-\chi^{*}\right\rangle \\
& =\left\langle\theta-P_{E P(f, \mathcal{K})}(\theta), x-P_{E P(f, \mathcal{K})}(\theta)\right\rangle \leq 0 .
\end{aligned}
$$

By using expressions (3.18), (3.20), (3.24) and Lemma 2.5, we obtain the required result.

Case 2. Suppose that there exists a subsequence $\left\{n_{i}\right\}$ of $\{n\}$ such that

$$
\left\|x_{n_{i}}-x^{*}\right\| \leq\left\|x_{n_{i+1}}-x^{*}\right\|, \quad \forall i \in \mathbb{N} .
$$

From Lemma 2.6, there is a sequence $\left\{m_{k}\right\} \subset \mathbb{N}$ with $\left\{m_{k}\right\} \rightarrow \infty$, such that

$$
\left\|x_{m_{k}}-x^{*}\right\| \leq\left\|x_{m_{k+1}}-x^{*}\right\| \quad \text { and } \quad\left\|x_{k}-x^{*}\right\| \leq\left\|x_{m_{k+1}}-x^{*}\right\|, \quad \text { for all } k \in \mathbb{N} \text {. }
$$

From expression (3.19) (for all $m_{k} \geq 2$ ), we have

$$
\begin{aligned}
& (1-\sigma)\left\|z_{m_{k}}-x_{m_{k}}\right\|^{2}+\sigma\left(1-2 c_{1} \sigma\right)\left\|x_{m_{k}}-y_{m_{k}}\right\|^{2}+\sigma\left(1-2 c_{2} \sigma\right)\left\|z_{m_{k}}-y_{m_{k}}\right\|^{2} \\
& \quad \leq\left\|x_{m_{k}}-x^{*}\right\|^{2}-\left\|x_{m_{k}+1}-x^{*}\right\|^{2}+\alpha_{m_{k}}\left\|x^{*}\right\|^{2}+\alpha_{m_{k}} A_{0} .
\end{aligned}
$$

The aforementioned expression implies that

$$
\lim _{k \rightarrow \infty}\left\|z_{m_{k}}-x_{m_{k}}\right\|=\lim _{k \rightarrow \infty}\left\|x_{m_{k}}-y_{m_{k}}\right\|=\lim _{k \rightarrow \infty}\left\|z_{m_{k}}-y_{m_{k}}\right\|=0 .
$$

Similar to expression (3.24), we have

$$
\limsup _{k \rightarrow \infty}\left\langle-\chi^{*}, x_{m_{k}}-x^{*}\right\rangle \leq 0 \text {. }
$$


From expression (3.18), we obtain

$$
\begin{aligned}
\left\|x_{m_{k}+1}-x^{*}\right\|^{2} \leq & \left(1-\alpha_{m_{k}} \delta_{m_{k}}\right)\left\|x_{m_{k}}-x^{*}\right\|^{2}+2 \alpha_{m_{k}} \delta_{m_{k}}\left(1-\alpha_{m_{k}}\right)\left\langle z_{m_{k}}-x_{m_{k}},\left(1-\alpha_{m_{k}} \delta_{m_{k}}\right) q_{m_{k}}\right. \\
& \left.+\alpha_{m_{k}} \delta_{m_{k}}\left(1-\alpha_{m_{k}}\right)\left(z_{m_{k}}-x_{m_{k}}\right)-x^{*}\right\rangle+2 \alpha_{m_{k}} \delta_{m_{k}}\left(1-\alpha_{m_{k}}\right)\left\langle-x^{*}, z_{m_{k}}-x_{m_{k}}\right\rangle \\
& +2 \alpha_{m_{k}} \delta_{m_{k}}\left\langle-x^{*}, x_{m_{k}}-x^{*}\right\rangle+2 \alpha_{m_{k}}^{2} \delta_{m_{k}}^{2}\left\langle x^{*}, x_{m_{k}}\right\rangle .
\end{aligned}
$$

It is given that $\left\|x_{m_{k}}-x^{*}\right\| \leq\left\|x_{m_{k+1}}-x^{*}\right\|$. Thus, we have

$$
\begin{aligned}
\left\|x_{m_{k}+1}-x^{*}\right\|^{2} \leq & \left(1-\alpha_{m_{k}} \delta_{m_{k}}\right)\left\|x_{m_{k+1}}-x^{*}\right\|^{2}+2 \alpha_{m_{k}} \delta_{m_{k}}\left(1-\alpha_{m_{k}}\right)\left\langle z_{m_{k}}-x_{m_{k}},\left(1-\alpha_{m_{k}} \delta_{m_{k}}\right) q_{m_{k}}\right. \\
& \left.+\alpha_{m_{k}} \delta_{m_{k}}\left(1-\alpha_{m_{k}}\right)\left(z_{m_{k}}-x_{m_{k}}\right)-x^{*}\right\rangle+2 \alpha_{m_{k}} \delta_{m_{k}}\left(1-\alpha_{m_{k}}\right)\left\langle-x^{*}, z_{m_{k}}-x_{m_{k}}\right\rangle \\
& +2 \alpha_{m_{k}} \delta_{m_{k}}\left\langle-x^{*}, x_{m_{k}}-x^{*}\right\rangle+2 \alpha_{m_{k}}^{2} \delta_{m_{k}}^{2}\left\langle x^{*}, x_{m_{k}}\right\rangle .
\end{aligned}
$$

Expressions (3.25) and (3.30) imply that

$$
\begin{aligned}
\left\|x_{k}-x^{*}\right\|^{2} \leq & \left\|x_{m_{k}+1}-x^{*}\right\|^{2} \\
\leq & 2\left(1-\alpha_{m_{k}}\right)\left\langle z_{m_{k}}-x_{m_{k}},\left(1-\alpha_{m_{k}} \delta_{m_{k}}\right) q_{m_{k}}+\alpha_{m_{k}} \delta_{m_{k}}\left(1-\alpha_{m_{k}}\right)\left(z_{m_{k}}-x_{m_{k}}\right)-x^{*}\right\rangle \\
& +2\left(1-\alpha_{m_{k}}\right)\left\langle-x^{*}, z_{m_{k}}-x_{m_{k}}\right\rangle+2\left\langle-x^{*}, x_{m_{k}}-x^{*}\right\rangle+2 \alpha_{m_{k}} \delta_{m_{k}}\left\langle x^{*}, x_{m_{k}}\right\rangle, \quad \forall n \geq 2 .
\end{aligned}
$$

Since $\alpha_{m_{k}} \rightarrow 0$, it follows from (3.27) such that

$$
\lim _{n \rightarrow \infty}\left\|x_{k}-x^{*}\right\|^{2} \leq \lim _{n \rightarrow \infty}\left\|x_{m_{k}+1}-x^{*}\right\|^{2} \leq 0 .
$$

Consequently, $x_{n} \rightarrow x^{*}$. This completes the proof.

\section{Modified explicit subgradient extragradient method and its convergence analysis}

The following is the second method in detail.

\section{Algorithm 2}

Initialization: Let $x_{1} \in \mathcal{E}, \sigma<\min \left\{1, \frac{1}{2 c_{1}}, \frac{1}{2 c_{2}}\right\}, \mu \in(0, \sigma), \zeta_{1}>0, \delta_{n} \subset[\delta, 1)$ with $0<\delta<1$ and $\alpha_{n}, \beta_{n} \subset(0,1)$ such that

$$
\lim _{n \rightarrow \infty} \alpha_{n}=0, \quad \sum_{n=1}^{\infty} \alpha_{n}=\infty \quad \text { and } \quad \liminf _{n \rightarrow \infty} \beta_{n}\left(1-\beta_{n}\right)>0
$$

Step 1: Compute

$$
y_{n}=\underset{y \in \mathcal{K}}{\arg \min }\left\{\zeta_{n} f\left(P_{\mathcal{K}}\left(x_{n}\right), y\right)+\frac{1}{2}\left\|P_{\mathcal{K}}\left(x_{n}\right)-y\right\|^{2}\right\}
$$

If $x_{n}=y_{n}$, then STOP. Otherwise, go to the next step.

Step 2: Construct a half space first

$$
\mathcal{E}_{n}=\left\{z \in \mathcal{E}:\left\langle P_{\mathcal{K}}\left(x_{n}\right)-\zeta_{n} \omega_{n}-y_{n}, z-y_{n}\right\rangle \leq 0\right\},
$$

where $\omega_{n} \in \partial f\left(P_{\mathcal{K}}\left(x_{n}\right), y_{n}\right)$ and solve the following convex problem:

$$
z_{n}=\underset{y \in \mathcal{E}_{n}}{\arg \min }\left\{\mu \zeta_{n} f\left(y_{n}, y\right)+\frac{1}{2}\left\|P_{\mathcal{K}}\left(x_{n}\right)-y\right\|^{2}\right\} .
$$


Step 3: Compute

$$
x_{n+1}=\alpha_{n}\left(1-\delta_{n}\right) x_{n}+\left(1-\alpha_{n}\right)\left[\beta_{n} z_{n}+\left(1-\beta_{n}\right) x_{n}\right]
$$

Step 4: Revising the step size as follows:

$$
\zeta_{n+1}=\left\{\begin{array}{c}
\min \left\{\sigma, \frac{\mu f\left(y_{n}, z_{n}\right)}{f\left(P_{\mathcal{K}}\left(x_{n}\right), z_{n}\right)-f\left(P_{\mathcal{K}}\left(x_{n}\right), y_{n}\right)-c_{1}\left\|P_{\mathcal{K}}\left(x_{n}\right)-y_{n}\right\|^{2}-c_{2}\left\|z_{n}-y_{n}\right\|^{2}+1}\right\}, \\
\quad \text { if } \frac{\mu f\left(y_{n}, z_{n}\right)}{f\left(P_{\mathcal{K}}\left(x_{n}\right), z_{n}\right)-f\left(P_{\mathcal{K}}\left(x_{n}\right), y_{n}\right)-c_{1}\left\|P_{\mathcal{K}}\left(x_{n}\right)-y_{n}\right\|^{2}-c_{2}\left\|z_{n}-y_{n}\right\|^{2}+1}>0, \\
\quad \text { else. }
\end{array}\right.
$$

Set $n:=n+1$ and go back to Step 1 .

Remark 4.1. The iteration $\zeta_{n+1}$ in (4.1) is well-defined and

$$
\zeta_{n+1}\left(f\left(P_{\mathcal{K}}\left(x_{n}\right), z_{n}\right)-f\left(P_{\mathcal{K}}\left(x_{n}\right), y_{n}\right)-c_{1}\left\|P_{\mathcal{K}}\left(x_{n}\right)-y_{n}\right\|^{2}-c_{2}\left\|y_{n}-z_{n}\right\|^{2}\right) \leq \mu f\left(y_{n}, z_{n}\right)
$$

Lemma 4.2. Let $f: \mathcal{E} \times \mathcal{E} \rightarrow \mathbb{R}$ be a bi-function satisfying conditions (C1)-(C4). Then, for each $x^{*} \in E P(f, \mathcal{K}) \neq \varnothing$, we have

$\left\|z_{n}-x^{*}\right\|^{2} \leq\left\|P_{\mathcal{K}}\left(x_{n}\right)-x^{*}\right\|^{2}-\left(1-\zeta_{n+1}\right)\left\|z_{n}-P_{\mathcal{K}}\left(x_{n}\right)\right\|^{2}-\zeta_{n+1}\left(1-2 c_{1} \zeta_{n}\right)\left\|P_{\mathcal{K}}\left(x_{n}\right)-y_{n}\right\|^{2}-\zeta_{n+1}\left(1-2 c_{2} \zeta_{n}\right)\left\|z_{n}-y_{n}\right\|^{2}$.

Theorem 4.3. Let $\left\{x_{n}\right\}$ be a sequence generated by Algorithm 2 and the solution set EP( $\left.f, \mathcal{K}\right)$ is non-empty. Then, the sequence $\left\{x_{n}\right\}$ is strongly convergent to an element $x^{*} \in \operatorname{EP}(f, \mathcal{K})$.

Proof. First, we need to show that $\left\{x_{n}\right\}$ is a bounded sequence. By the use of Lemma 4.2, we have

$$
\begin{aligned}
\left\|x_{n+1}-x^{*}\right\|^{2}= & \left\|\alpha_{n}\left(1-\delta_{n}\right) x_{n}+\left(1-\alpha_{n}\right)\left[\beta_{n} z_{n}+\left(1-\beta_{n}\right) x_{n}\right]-x^{*}\right\|^{2} \\
= & \left\|\alpha_{n}\left[\left(1-\delta_{n}\right) x_{n}-x^{*}\right]+\left(1-\alpha_{n}\right)\left[\beta_{n}\left(z_{n}-x^{*}\right)+\left(1-\beta_{n}\right)\left(x_{n}-x^{*}\right)\right]\right\|^{2} \\
\leq & \alpha_{n}\left\|\left(1-\delta_{n}\right) x_{n}-x^{*}\right\|^{2}+\left(1-\alpha_{n}\right)\left\|\beta_{n}\left(z_{n}-x^{*}\right)+\left(1-\beta_{n}\right)\left(x_{n}-x^{*}\right)\right\|^{2} \\
\leq & \alpha_{n}\left\|\left(1-\delta_{n}\right)\left(x_{n}-x^{*}\right)+\delta_{n} x^{*}\right\|^{2}+\left(1-\alpha_{n}\right)\left[\beta_{n}\left\|z_{n}-x^{*}\right\|^{2}+\left(1-\beta_{n}\right)\left\|x_{n}-x^{*}\right\|^{2}\right. \\
& \left.-\beta_{n}\left(1-\beta_{n}\right)\left\|z_{n}-x_{n}\right\|^{2}\right] \\
\leq & \alpha_{n}\left[\left(1-\delta_{n}\right)\left\|x_{n}-x^{*}\right\|^{2}+\delta_{n}\left\|x^{*}\right\|^{2}\right]+\left(1-\alpha_{n}\right)\left[\left\|x_{n}-x^{*}\right\|^{2}-\beta_{n}\left(1-\zeta_{n+1}\right)\left\|z_{n}-P_{\mathcal{K}}\left(x_{n}\right)\right\|^{2}\right. \\
& \left.-\beta_{n} \zeta_{n+1}\left(1-2 c_{1} \zeta_{n}\right)\left\|P_{\mathcal{K}}\left(x_{n}\right)-y_{n}\right\|^{2}-\beta_{n} \zeta_{n+1}\left(1-2 c_{2} \zeta_{n}\right)\left\|z_{n}-y_{n}\right\|^{2}-\beta_{n}\left(1-\beta_{n}\right)\left\|z_{n}-x_{n}\right\|^{2}\right] \\
\leq & \left(1-\alpha_{n} \delta_{n}\right)\left\|x_{n}-x^{*}\right\|^{2}+\alpha_{n} \delta_{n}\left\|x^{*}\right\|^{2}-\left(1-\alpha_{n}\right)\left[\beta_{n}(1-\sigma)\left\|z_{n}-P_{\mathcal{K}}\left(x_{n}\right)\right\|^{2}\right. \\
& \left.+\beta_{n} \sigma\left(1-2 c_{1} \sigma\right)\left\|P_{\mathcal{K}}\left(x_{n}\right)-y_{n}\right\|^{2}+\beta_{n} \sigma\left(1-2 c_{2} \sigma\right)\left\|z_{n}-y_{n}\right\|^{2}+\beta_{n}\left(1-\beta_{n}\right)\left\|z_{n}-x_{n}\right\|^{2}\right] .
\end{aligned}
$$

Since $0<\sigma<1$, the above inequality implies that

$$
\left\|x_{n+1}-x^{*}\right\|^{2} \leq \max \left\{\left\|x_{n}-x^{*}\right\|^{2},\left\|x^{*}\right\|^{2}\right\} \leq \max \left\{\left\|x_{2}-x^{*}\right\|^{2},\left\|x^{*}\right\|^{2}\right\} .
$$

It implies that $\left\{x_{n}\right\}$ is a bounded sequence. By using $x_{n+1}$ with Lemma 2.4 gives that (see equation (3.17) [31]):

$$
\left\|x_{n+1}-x^{*}\right\|^{2} \leq\left(1-\alpha_{n} \delta_{n}\right)\left\|x_{n}-x^{*}\right\|^{2}+2 \alpha_{n} \delta_{n}\left(1-\alpha_{n}\right) \beta_{n}\left\langle z_{n}-x_{n}, x_{n+1}-x^{*}\right\rangle+2 \alpha_{n} \delta_{n}\left\langle-x^{*}, x_{n+1}-x^{*}\right\rangle .
$$

Case 1. Assume that there is an $m_{2} \in \mathbb{N}\left(m_{2} \geq 2\right)$ such that

$$
\left\|x_{n+1}-x^{*}\right\| \leq\left\|x_{n}-x^{*}\right\|, \quad \forall n \geq m_{2} .
$$


Then, the $\lim _{n \rightarrow \infty}\left\|x_{n}-x^{*}\right\|$ exists. By expression (4.3), we have

$$
\begin{aligned}
& \beta_{n}\left[(1-\sigma)\left\|z_{n}-P_{\mathcal{K}}\left(x_{n}\right)\right\|^{2}+\sigma\left(1-2 c_{1} \sigma\right)\left\|P_{\mathcal{K}}\left(x_{n}\right)-y_{n}\right\|^{2}+\sigma\left(1-2 c_{2} \sigma\right)\left\|z_{n}-y_{n}\right\|^{2}+\left(1-\beta_{n}\right)\left\|z_{n}-x_{n}\right\|^{2}\right] \\
& \quad \leq\left\|x_{n}-x^{*}\right\|^{2}-\left\|x_{n+1}-x^{*}\right\|^{2}+\alpha_{n}\left\|x^{*}\right\|^{2}+\alpha_{n} B_{0},
\end{aligned}
$$

where $B_{0}$ is the finite number

$$
\begin{aligned}
B_{0}= & \sup \left\{\beta _ { n } \left[(1-\sigma)\left\|z_{n}-P_{\mathcal{K}}\left(x_{n}\right)\right\|^{2}+\sigma\left(1-2 c_{1} \sigma\right)\left\|P_{\mathcal{K}}\left(x_{n}\right)-y_{n}\right\|^{2}+\sigma\left(1-2 c_{2} \sigma\right)\left\|z_{n}-y_{n}\right\|^{2}\right.\right. \\
& \left.\left.+\left(1-\beta_{n}\right)\left\|z_{n}-x_{n}\right\|^{2}\right]: \forall n \in \mathbb{N}\right\} .
\end{aligned}
$$

Thus, expression (4.7) implies that

$$
\lim _{n \rightarrow \infty}\left\|P_{\mathcal{K}}\left(x_{n}\right)-z_{n}\right\|=\lim _{n \rightarrow \infty}\left\|P_{\mathcal{K}}\left(x_{n}\right)-y_{n}\right\|=\lim _{n \rightarrow \infty}\left\|z_{n}-y_{n}\right\|=0=\lim _{n \rightarrow \infty}\left\|x_{n}-z_{n}\right\|=0 .
$$

Due to the boundedness of the sequence $\left\{x_{n}\right\}$, there exists a subsequence $\left\{x_{n_{k}}\right\}$ of $\left\{x_{n}\right\}$ such that $\left\{x_{n_{k}}\right\}$ weakly converges to $x \in \mathcal{K}$ and

$$
\limsup _{n \rightarrow \infty}\left\langle-x^{*}, x_{n}-x^{*}\right\rangle=\limsup _{k \rightarrow \infty}\left\langle-x^{*}, x_{n_{k}}-x^{*}\right\rangle=\left\langle-x^{*}, x-x^{*}\right\rangle .
$$

Similar to expression (3.5), we have

$$
\mu \zeta_{n_{k}} f\left(y_{n_{k}}, y\right)-\mu \zeta_{n_{k}} f\left(y_{n_{k}}, z_{n_{k}}\right) \geq\left\langle P_{\mathcal{K}}\left(x_{n_{k}}\right)-z_{n_{k}}, y-z_{n_{k}}\right\rangle, \quad \forall y \in \mathcal{E}_{n}
$$

By letting $k \rightarrow+\infty$, we have

$$
f(x, y) \geq 0, \quad \forall y \in \mathcal{K} .
$$

It implies that $x \in E P(f, \mathcal{K})$. By expression (4.10) and Lemma 2.3, we obtain

$$
\begin{aligned}
\limsup _{n \rightarrow \infty}\left\langle-x^{*}, x_{n}-x^{*}\right\rangle & =\limsup _{k \rightarrow \infty}\left\langle-x^{*}, x_{n_{k}}-x^{*}\right\rangle=\left\langle-x^{*}, x-x^{*}\right\rangle \\
& =\left\langle\theta-P_{E P(f, \mathcal{K})}(\theta), x-P_{E P(f, \mathcal{K})}(\theta)\right\rangle \leq 0 .
\end{aligned}
$$

By expression (4.5) and Lemma 2.5, we obtain the required result.

Case 2. Suppose that there exists a subsequence $\left\{n_{i}\right\}$ of $\{n\}$ such that

$$
\left\|x_{n_{i}}-x^{*}\right\| \leq\left\|x_{n_{i+1}}-x^{*}\right\|, \quad \forall i \in \mathbb{N} .
$$

Then, by Lemma 2.6 there is a non-decreasing sequence $\left\{m_{k}\right\} \subset \mathbb{N}$ with $m_{k} \rightarrow \infty$, which implies that

$$
\left\|x_{m_{k}}-x^{*}\right\| \leq\left\|x_{m_{k+1}}-x^{*}\right\| \text { and }\left\|x_{k}-x^{*}\right\| \leq\left\|x_{m_{k+1}}-x^{*}\right\|, \quad \text { for all } k \in \mathbb{N} .
$$

From expression (4.5), we obtain

$$
\begin{aligned}
\left\|x_{m_{k}+1}-x^{*}\right\|^{2} \leq & \left(1-\alpha_{m_{k}} \delta_{m_{k}}\right)\left\|x_{m_{k}}-x^{*}\right\|^{2}+2 \alpha_{m_{k}} \delta_{m_{k}}\left(1-\alpha_{m_{k}}\right) \beta_{m_{k}}\left\langle z_{m_{k}}-x_{m_{k}}, x_{m_{k}+1}-x^{*}\right\rangle \\
& +2 \alpha_{m_{k}} \delta_{m_{k}}\left\langle-x^{*}, x_{m_{k}+1}-x^{*}\right\rangle .
\end{aligned}
$$

The remaining part is similar to Case 2 in Theorem 3.3. This completes the proof.

\section{Applications}

Now, we study the applications of our main results to solve fixed point problems. An operator $T: \mathcal{K} \subset \mathcal{E} \rightarrow \mathcal{K}$ is said to be

(i) $\kappa$-strict pseudocontraction [39] on $\mathcal{K}$ if

$$
\left\|T y_{1}-T y_{2}\right\|^{2} \leq\left\|y_{1}-y_{2}\right\|^{2}+\kappa\left\|\left(y_{1}-T y_{1}\right)-\left(y_{2}-T y_{2}\right)\right\|^{2}, \quad \forall y_{1}, y_{2} \in \mathcal{K},
$$


which is equivalent to

$$
\left\langle T y_{1}-T y_{2}, y_{1}-y_{2}\right\rangle \leq\left\|y_{1}-y_{2}\right\|^{2}-\frac{1-\kappa}{2}\left\|\left(y_{1}-T y_{1}\right)-\left(y_{2}-T y_{2}\right)\right\|^{2}, \quad \forall y_{1}, y_{2} \in \mathcal{K} .
$$

(ii) Weakly sequentially continuous on $\mathcal{K}$ if

$$
T\left(x_{n}\right) \rightarrow T(p) \text { for any sequence in } \mathcal{K} \text { satisfying } x_{n} \rightarrow p .
$$

Corollary 5.1. Assume that $\mathcal{K}$ is a non-empty, convex and closed subset of a Hilbert space $\mathcal{E}$ and $T: \mathcal{K} \rightarrow \mathcal{K}$ is a $\kappa$-strict pseudo-contraction and weakly continuous with $\operatorname{Fix}(T) \neq \varnothing$. Choose $x_{1} \in \mathcal{K}, \sigma<\min \left\{1, \frac{1-\kappa}{3-2 \kappa}\right\}$, $\mu \in(0, \sigma), \zeta_{1}>0, \delta_{n} \subset[\delta, 1)$ with $0<\delta<1$ and $\alpha_{n} \subset(0,1)$ such that

$$
\lim _{n \rightarrow \infty} \alpha_{n}=0 \text { and } \sum_{n=1}^{\infty} \alpha_{n}=\infty
$$

Compute $x_{n+1}=P_{\mathcal{K}}\left[\alpha_{n} x_{n}+\left(1-\alpha_{n}\right) z_{n}-\alpha_{n} \delta_{n} x_{n}\right]$, where

$$
\left\{\begin{array}{l}
y_{n}=\left(1-\zeta_{n}\right) x_{n}+\zeta_{n} T\left(x_{n}\right), \\
z_{n}=P_{\mathcal{E}_{n}}\left[x_{n}-\mu \zeta_{n}\left(y_{n}-T\left(y_{n}\right)\right)\right]
\end{array}\right.
$$

where $\mathcal{E}_{n}=\left\{z \in \mathcal{E}:\left\langle\left(1-\zeta_{n}\right) x_{n}+\zeta_{n} T\left(x_{n}\right)-y_{n}, z-y_{n}\right\rangle \leq 0\right\}$. Compute

$$
\zeta_{n+1}=\left\{\begin{array}{l}
\min \left\{\sigma, \frac{\mu\left\langle y_{n}-T y_{n}, z_{n}-y_{n}\right\rangle}{\left\langle x_{n}-T\left(x_{n}\right), z_{n}-y_{n}\right\rangle-\left(\frac{3-2 k}{2-2 \kappa}\right)\left\|x_{n}-y_{n}\right\|^{2}-\left(\frac{3-2 \kappa}{2-2 \kappa}\right)\left\|z_{n}-y_{n}\right\|^{2}+1}\right\}, \\
\text { if } \quad \frac{\mu\left\langle y_{n}-T y_{n}, z_{n}-y_{n}\right\rangle}{\left\langle x_{n}-T\left(x_{n}\right), z_{n}-y_{n}\right\rangle-\left(\frac{3-2 \kappa}{2-2 \kappa}\right)\left\|x_{n}-y_{n}\right\|^{2}-\left(\frac{3-2 \kappa}{2-2 \kappa}\right)\left\|z_{n}-y_{n}\right\|^{2}+1}>0, \\
\sigma \quad \text { else. }
\end{array}\right.
$$

Then, the sequence $\left\{x_{n}\right\}$ converges strongly to an element $x^{*}=P_{F i x(T)}(\theta)$.

Corollary 5.2. Assume that $\mathcal{K}$ is a non-empty, convex and closed subset of a Hilbert space $\mathcal{E}$ with $T: \mathcal{K} \rightarrow \mathcal{K}$ is a $\kappa$-strict pseudocontraction and weakly continuous with $\operatorname{Fix}(T) \neq \varnothing$. Choose $x_{1} \in \mathcal{E}, \sigma<\min \left\{1, \frac{1-\kappa}{3-2 \kappa}\right\}$, $\mu \in(0, \sigma), \zeta_{1}>0, \delta_{n} \subset[\delta, 1)$ with $0<\delta<1$ and $\alpha_{n}, \beta_{n} \subset(0,1)$ such that

$$
\lim _{n \rightarrow \infty} \alpha_{n}=0, \quad \sum_{n=1}^{\infty} \alpha_{n}=\infty \text { and } \liminf _{n \rightarrow \infty} \beta_{n}\left(1-\beta_{n}\right)>0 .
$$

Compute $x_{n+1}=\alpha_{n}\left(1-\delta_{n}\right) x_{n}+\left(1-\alpha_{n}\right)\left[\beta_{n} z_{n}+\left(1-\beta_{n}\right) x_{n}\right]$, where

$$
\left\{\begin{array}{l}
y_{n}=\left(1-\zeta_{n}\right) P_{\mathcal{K}}\left(x_{n}\right)+\zeta_{n} T\left(P_{\mathcal{K}}\left(x_{n}\right)\right), \\
z_{n}=P_{\mathcal{E}_{n}}\left[x_{n}-\mu \zeta_{n}\left(y_{n}-T\left(y_{n}\right)\right)\right],
\end{array}\right.
$$

where $\mathcal{E}_{n}=\left\{z \in \mathcal{E}:\left\langle\left(1-\zeta_{n}\right) P_{\mathcal{K}}\left(x_{n}\right)+\zeta_{n} T\left(P_{\mathcal{K}}\left(x_{n}\right)\right)-y_{n}, z-y_{n}\right\rangle \leq 0\right\}$. Compute

$$
\zeta_{n+1}=\left\{\begin{array}{c}
\min \left\{\sigma, \frac{\mu\left\langle y_{n}-T y_{n}, z_{n}-y_{n}\right\rangle}{\left\langle P_{\mathcal{K}}\left(x_{n}\right)-T P_{\mathcal{K}}\left(x_{n}\right), z_{n}-y_{n}\right\rangle-\left(\frac{3-2 k}{2-2 \kappa}\right)\left\|P_{\mathcal{K}}\left(x_{n}\right)-y_{n}\right\|^{2}-\left(\frac{3-2 \kappa}{2-2 \kappa}\right)\left\|z_{n}-y_{n}\right\|^{2}+1}\right\}, \\
\text { if } \frac{\mu\left\langle y_{n}-T y_{n}, z_{n}-y_{n}\right\rangle}{\left\langle P_{\mathcal{K}}\left(x_{n}\right)-T P_{\mathcal{K}}\left(x_{n}\right), z_{n}-y_{n}\right\rangle-\left(\frac{3-2 \kappa}{2-2 \kappa}\right)\left\|P_{\mathcal{K}}\left(x_{n}\right)-y_{n}\right\|^{2}-\left(\frac{3-2 \kappa}{2-2 \kappa}\right)\left\|z_{n}-y_{n}\right\|^{2}+1}>0, \\
\sigma \quad \text { else. }
\end{array}\right.
$$

Then, the sequence $\left\{x_{n}\right\}$ converges strongly to an element $x^{*}=P_{F i x(T)}(\theta)$. 
The variational inequality problem is defined in the following way:

$$
\text { Find } x^{*} \in \mathcal{K} \text { such that }\left\langle G\left(x^{*}\right), y-x^{*}\right\rangle \geq 0, \quad \forall y \in \mathcal{K} \text {. }
$$

Note: If $f(x, y):=\langle G(x), y-x\rangle$ for all $x, y \in \mathcal{K}$, the EP transforms into variational inequality problem with $L=2 c_{1}=2 c_{2}$ (for details see [40]). Moreover, we have

$$
\left\{\begin{array}{l}
y_{n}=\underset{y \in \mathcal{K}}{\arg \min }\left\{\zeta_{n} f\left(x_{n}, y\right)+\frac{1}{2}\left\|x_{n}-y\right\|^{2}\right\}=P_{\mathcal{K}}\left[x_{n}-\zeta_{n} G\left(x_{n}\right)\right], \\
z_{n}=\underset{y \in \mathcal{E}_{n}}{\arg \min }\left\{\mu \zeta_{n} f\left(y_{n}, y\right)+\frac{1}{2}\left\|x_{n}-y\right\|^{2}\right\}=P_{\mathcal{E}_{n}}\left[x_{n}-\mu \zeta_{n} G\left(y_{n}\right)\right] .
\end{array}\right.
$$

Suppose that $G$ meets the following conditions:

(G1) $G$ is pseudomonotone on $\mathcal{K}$ with $\operatorname{VI}(G, \mathcal{K})$ is non-empty;

(G2) $G$ satisfies $L$-Lipschitz continuity on $\mathcal{K}$ through $L>0$;

(G3) $\lim \sup _{n \rightarrow \infty}\left\langle G\left(x_{n}\right), y-x_{n}\right\rangle \leq\langle G(p), y-p\rangle$ for every $y \in \mathcal{K}$ and $\left\{x_{n}\right\} \subset \mathcal{K}$ satisfying $x_{n} \rightarrow p$.

Corollary 5.3. Let a mapping $G: \mathcal{K} \rightarrow \mathcal{E}$ satisfy conditions (G1)-(G3). Assume that sequence $\left\{x_{n}\right\}$ is generated as follows:

(i) Let $x_{1} \in \mathcal{K}, \sigma<\min \left\{1, \frac{1}{L}\right\}, \mu \in(0, \sigma), \zeta_{1}>0, \delta_{n} \subset[\delta, 1)$ with $0<\delta<1$ and $\alpha_{n} \subset(0,1)$ such that

$$
\lim _{n \rightarrow \infty} \alpha_{n}=0 \text { and } \sum_{n=1}^{\infty} \alpha_{n}=\infty \text {. }
$$

(ii) Compute $x_{n+1}=P_{\mathcal{K}}\left[\alpha_{n} x_{n}+\left(1-\alpha_{n}\right) z_{n}-\alpha_{n} \delta_{n} x_{n}\right]$, where

$$
\left\{\begin{array}{l}
y_{n}=P_{\mathcal{K}}\left[x_{n}-\zeta_{n} G\left(x_{n}\right)\right], \\
z_{n}=P_{\mathcal{E}_{n}}\left[x_{n}-\mu \zeta_{n} G\left(y_{n}\right)\right],
\end{array}\right.
$$

where $\mathcal{E}_{n}=\left\{z \in \mathcal{E}:\left\langle x_{n}-\zeta_{n} G\left(x_{n}\right)-y_{n}, z-y_{n}\right\rangle \leq 0\right\}$. Compute

$$
\zeta_{n+1}=\left\{\begin{array}{c}
\min \left\{\sigma, \frac{\mu\left\langle G y_{n}, z_{n}-y_{n}\right\rangle}{\left\langle G x_{n}, z_{n}-y_{n}\right\rangle-\frac{L}{2}\left\|x_{n}-y_{n}\right\|^{2}-\frac{L}{2}\left\|z_{n}-y_{n}\right\|^{2}+1}\right\}, \\
\text { if } \frac{\mu\left\langle G y_{n}, z_{n}-y_{n}\right\rangle}{\left\langle G x_{n}, z_{n}-y_{n}\right\rangle-\frac{L}{2}\left\|x_{n}-y_{n}\right\|^{2}-\frac{L}{2}\left\|z_{n}-y_{n}\right\|^{2}+1}>0, \\
\sigma \quad \text { else. }
\end{array}\right.
$$

Then, the sequences $\left\{x_{n}\right\}$ strongly converge to a solution $x^{*} \in \operatorname{VI}(G, \mathcal{K})$.

Corollary 5.4. Let a mapping $G: \mathcal{K} \rightarrow \mathcal{E}$ satisfy conditions (G1)-(G3). Assume that sequence $\left\{x_{n}\right\}$ is generated as follows:

(i) Let $x_{1} \in \mathcal{E}, \sigma<\min \left\{1, \frac{1}{L}\right\}, \mu \in(0, \sigma), \zeta_{1}>0, \delta_{n} \subset[\delta, 1)$ with $0<\delta<1$ and $\alpha_{n}, \beta_{n} \subset(0,1)$ such that

$$
\lim _{n \rightarrow \infty} \alpha_{n}=0, \quad \sum_{n=1}^{\infty} \alpha_{n}=\infty \text { and } \liminf _{n \rightarrow \infty} \beta_{n}\left(1-\beta_{n}\right)>0 .
$$

(ii) Compute $x_{n+1}=\alpha_{n}\left(1-\delta_{n}\right) x_{n}+\left(1-\alpha_{n}\right)\left[\beta_{n} z_{n}+\left(1-\beta_{n}\right) x_{n}\right]$, where

$$
\left\{\begin{array}{l}
y_{n}=P_{\mathcal{K}}\left[P_{\mathcal{K}}\left(x_{n}\right)-\zeta_{n} G\left(P_{\mathcal{K}}\left(x_{n}\right)\right)\right], \\
z_{n}=P_{\mathcal{E}_{n}}\left[P_{\mathcal{K}}\left(x_{n}\right)-\mu \zeta_{n} G\left(y_{n}\right)\right],
\end{array}\right.
$$


where $\mathcal{E}_{n}=\left\{z \in \mathcal{E}:\left\langle P_{\mathcal{K}}\left(x_{n}\right)-\zeta_{n} G\left(P_{\mathcal{K}}\left(x_{n}\right)\right)-y_{n}, z-y_{n}\right\rangle \leq 0\right\}$. Compute

$$
\zeta_{n+1}=\left\{\begin{array}{c}
\min \left\{\sigma, \frac{\mu\left\langle G y_{n}, z_{n}-y_{n}\right\rangle}{\left\langle G\left(P_{\mathcal{K}}\left(x_{n}\right)\right), z_{n}-y_{n}\right\rangle-\frac{L}{2}\left\|P_{\mathcal{K}}\left(x_{n}\right)-y_{n}\right\|^{2}-\frac{L}{2}\left\|z_{n}-y_{n}\right\|^{2}+1}\right\}, \\
\text { if } \frac{\mu\left\langle G y_{n}, z_{n}-y_{n}\right\rangle}{\left\langle G\left(P_{\mathcal{K}}\left(x_{n}\right)\right), z_{n}-y_{n}\right\rangle-\frac{L}{2}\left\|P_{\mathcal{K}}\left(x_{n}\right)-y_{n}\right\|^{2}-\frac{L}{2}\left\|z_{n}-y_{n}\right\|^{2}+1}>0, \\
\sigma \quad \text { else. }
\end{array}\right.
$$

Then, the sequence $\left\{x_{n}\right\}$ strongly converges to a solution $x^{*} \in \operatorname{VI}(G, \mathcal{K})$.

\section{Numerical illustration}

The numerical discussion provided in this section demonstrates the efficiency of our proposed algorithms compared to Algorithms 3.1 and 3.2 in [31]. The MATLAB program was run on a PC (with Intel(R) Core(TM)i34010U CPU @ 1.70 GHz 1.70 GHz, RAM 4.00 GB) in MATLAB version 9.5 (R2018b). We use the built-in MATLAB fmincon function to solve minimization problems in all algorithms.

(i) The values for control parameters for Algorithm 3.1 (Alg3.1) and Algorithm 3.2 (Alg3.2) in [31] are $\alpha_{n}=\frac{1}{40 n}, \delta_{n}=\frac{1}{5}+\frac{1}{5 n}, \zeta_{n}=\frac{n}{7+2 c_{1} n}, \beta_{n}=\frac{1}{6}+\frac{1}{6 n}$ and $D_{n}=\left\|x_{n}-y_{n}\right\| \leq \epsilon$.

(ii) The values for control parameters for Algorithm 1 (Alg.1) and Algorithm 2 (Alg.2) are $\alpha_{n}=\frac{1}{40 n}$, $\delta_{n}=\frac{1}{5}+\frac{1}{5 n}, \beta_{n}=\frac{1}{6}+\frac{1}{6 n}, \mu=\frac{5}{13 c_{1}}, \zeta_{1}=\frac{1}{3 c_{1}}$ and $D_{n}=\left\|x_{n}-y_{n}\right\| \leq \epsilon$.

Example 6.1. Assume that $f: \mathcal{K} \times \mathcal{K} \rightarrow \mathbb{R}$ is

$$
f(x, y)=\sum_{i=2}^{5}\left(y_{i}-x_{i}\right)\|x\|, \quad \forall x, y \in \mathbb{R}^{5},
$$

where $\mathcal{K}=\left\{\left(x_{1}, \ldots, x_{5}\right): x_{1} \geq-1, x_{i} \geq 1, i=2, \ldots, 5\right\}$. Thus, bifunction $f$ is Lipschitz-type continuous through $c_{1}=c_{2}=2$ and meet the criterion $(\mathrm{C} 1)-(\mathrm{C} 4)$. The solution set of an $\operatorname{EP}$ is $\operatorname{EP}(f, \mathcal{K})=\left\{\left(x_{1}, 1,1,1,1\right): x_{1} \geq-1\right\}$ (see [31]). The numerical results are shown in Figures 1-4 and Table 1 and $\epsilon=10^{-4}$.
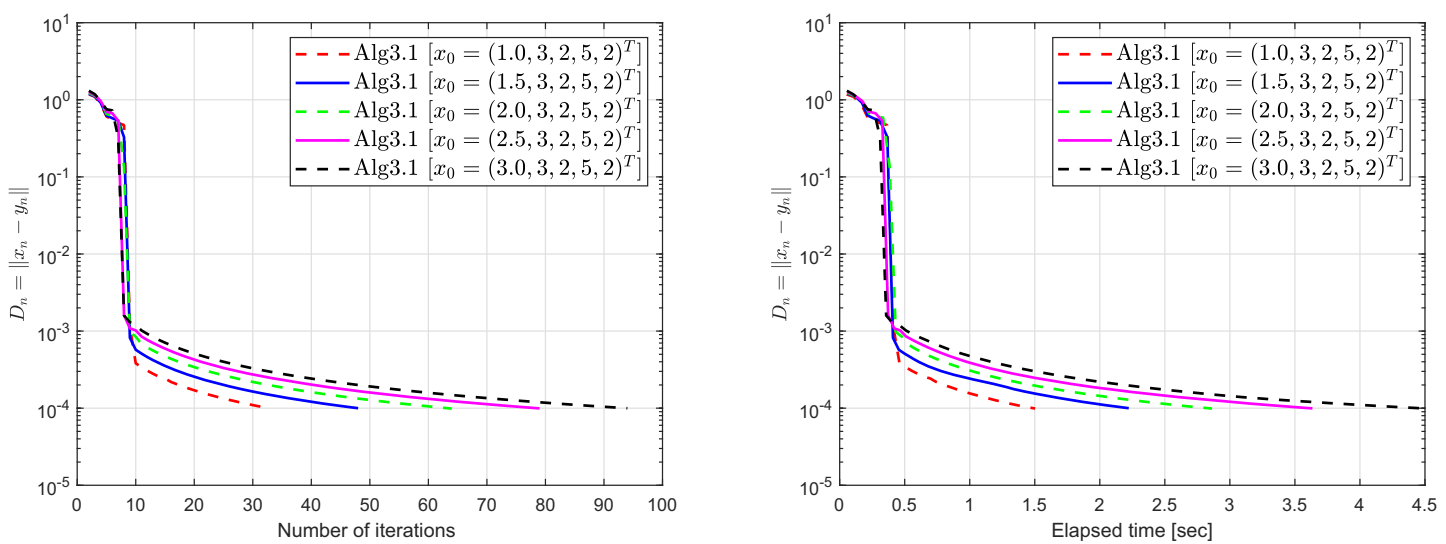

Figure 1: Example 6.1: Choosing different initial points and behavior for Algorithm 3.1 in [31]. 

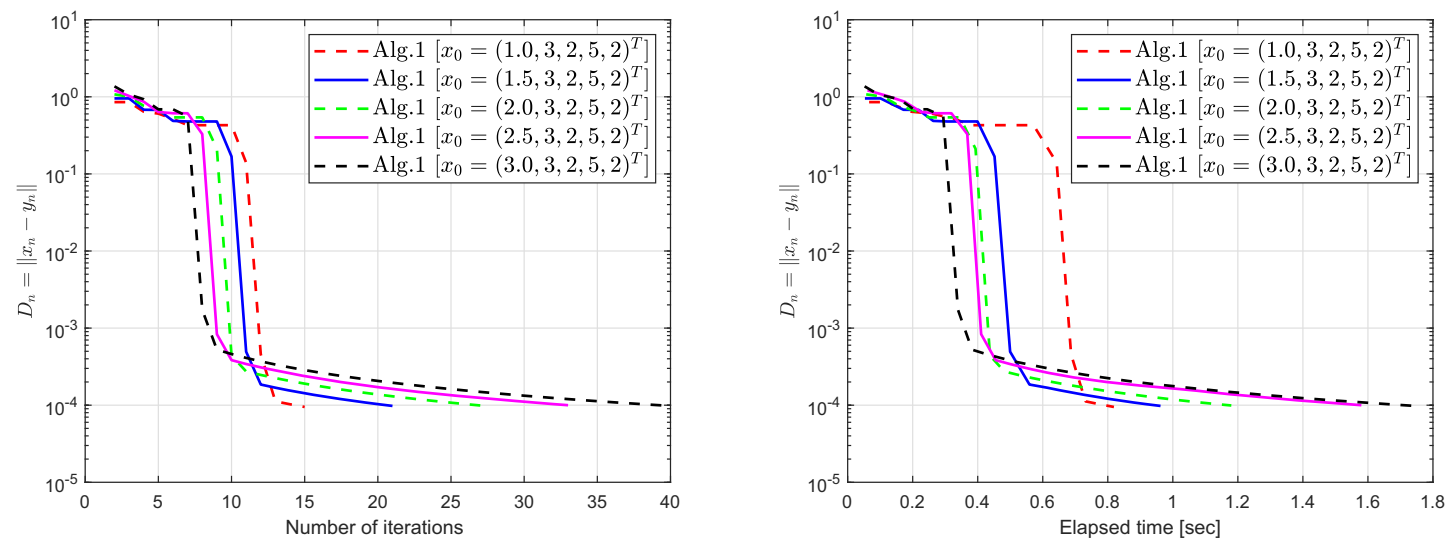

Figure 2: Example 6.1: Choosing different initial points and behavior for Algorithm 1.

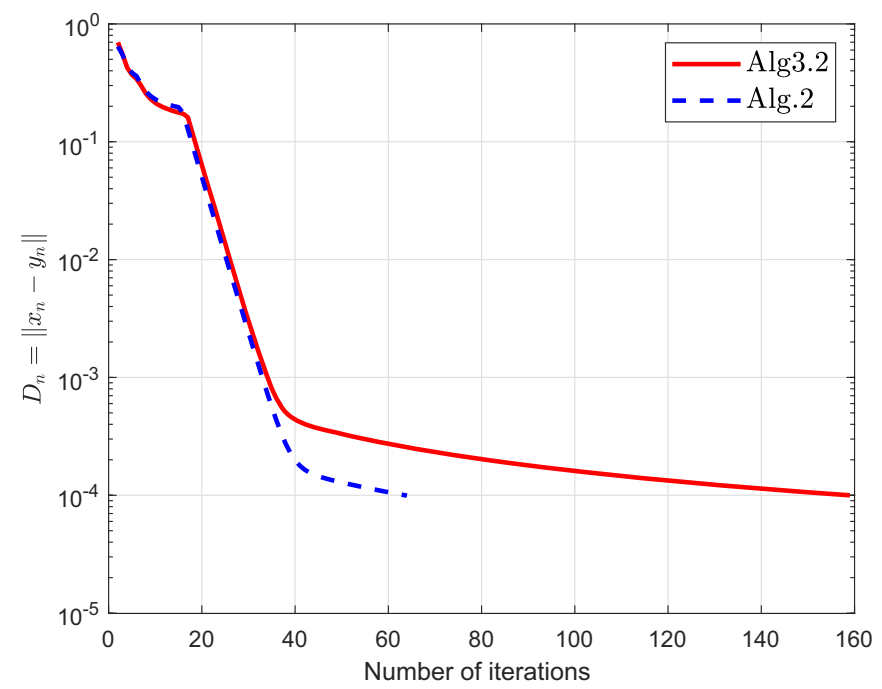

Figure 3: Example 6.1: Comparison of Algorithm 2 with Algorithm 3.2 in [31].

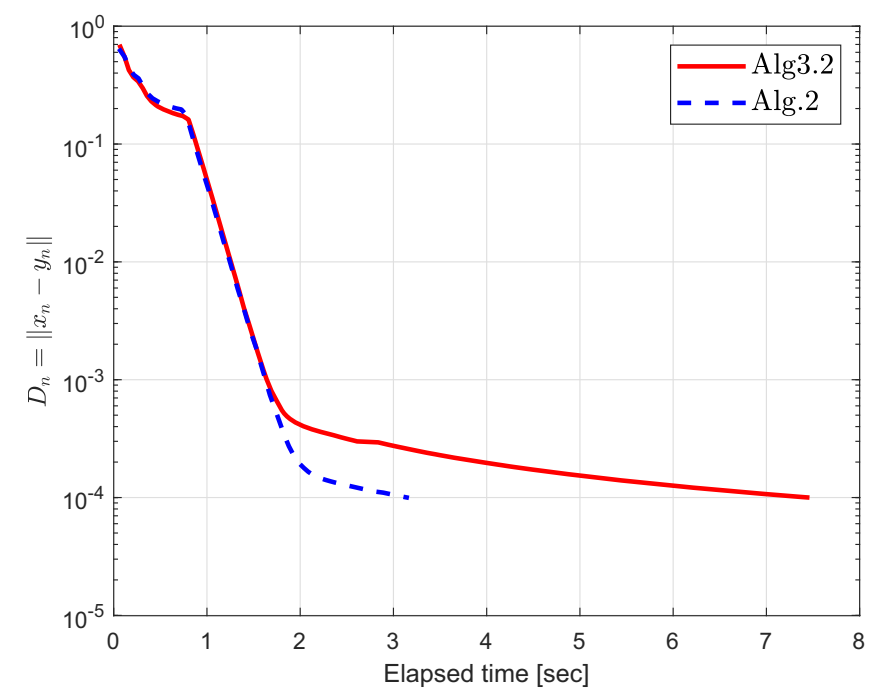

Figure 4: Example 6.1: Comparison of Algorithm 2 with Algorithm 3.2 in [31]. 
Table 1: Example 6.1: Numerical values of Algorithm 1 and Algorithm 3.1 in [31]

\begin{tabular}{|c|c|c|c|c|}
\hline \multirow[t]{2}{*}{$x_{1}$} & \multicolumn{2}{|c|}{ Number of iterations } & \multicolumn{2}{|c|}{ CPU time in seconds } \\
\hline & Alg3.1 & Alg.1 & Alg3.1 & Alg.1 \\
\hline$(1.0,3,2,5,2)^{T}$ & 33 & 15 & 1.5027 & 0.8189 \\
\hline$(1.5,3,2,5,2)^{T}$ & 48 & 21 & 2.2226 & 0.9622 \\
\hline$(2.0,3,2,5,2)^{T}$ & 64 & 27 & 2.8630 & 1.1799 \\
\hline$(2.5,3,2,5,2)^{T}$ & 79 & 33 & 3.6347 & 1.5886 \\
\hline$(3.0,3,2,5,2)^{T}$ & 94 & 40 & 4.4637 & 1.7434 \\
\hline
\end{tabular}

Example 6.2. Let there be $n$ firms that assemble the same commodity. Consider that a vector $x$ in which each element $x_{i}$ remains for the quantity of the substance produces by a firm $i$. We see the cost function $P$ as a decreasing affine function, which relies on the amount of $S=\sum_{i=1}^{m} x_{i}$, i.e., $P_{i}(S)=\alpha_{i}-\psi_{i} S$, where $\alpha_{i}>0$, $\psi_{i}>0$. The function of earnings of every firm $i$ is defined by $F_{i}(x)=P_{i}(S) x_{i}-t_{i}\left(x_{i}\right)$, where $t_{i}\left(x_{i}\right)$ is the tax value and cost for developing item $x_{i}$. Consider that $\mathcal{K}_{i}=\left[x_{i}^{\min }, x_{i}^{\max }\right]$ is the collections of measures related to any company $i$, and the game plan works out for the whole design and takes the form as $\mathcal{K}:=\mathcal{K}_{1} \times \mathcal{K}_{2} \times$ $\cdots \times \mathcal{K}_{n}$. Each company wants to attain its peak earnings by pursuing the respective stage of production on the premise that the performance of the other firms is an input parameter. The commonly used modelling methodology is based on the famous Nash equilibrium principle. We would like to point out that point $x^{*} \epsilon$ $\mathcal{K}=\mathcal{K}_{1} \times \mathcal{K}_{2} \times \cdots \times \mathcal{K}_{n}$ is the point of equilibrium of the model if $F_{i}\left(x^{*}\right) \geq F_{i}\left(x^{*}\left[x_{i}\right]\right), \forall x_{i} \in \mathcal{K}_{i}, \forall i=1,2, \ldots, n$, with the vector $x^{*}\left[x_{i}\right]$ representing the vector obtained from $x^{*}$ by taking $x_{i}^{*}$ with $x_{i}$. Furthermore, let $f(x, y):=\beta(x, y)-\beta(x, x)$ with $\beta(x, y):=-\sum_{i=1}^{n} F_{i}\left(x\left[y_{i}\right]\right)$, and the problem of getting the Nash equilibrium point of the model may be as follows:

Find $x^{*} \in \mathcal{K}: f\left(x^{*}, y\right) \geq 0, \forall y \in \mathcal{K}$.

It follows from the paper [29] that the bifunction $f$ could be taken in the following form:

$$
f(x, y)=\langle A x+B y+c, y-x\rangle,
$$

where $c \in \mathbb{R}^{5}$ and $A, B$ are

$$
A=\left(\begin{array}{ccccc}
3.1 & 2 & 0 & 0 & 0 \\
2 & 3.6 & 0 & 0 & 0 \\
0 & 0 & 3.5 & 2 & 0 \\
0 & 0 & 2 & 3.3 & 0 \\
0 & 0 & 0 & 0 & 3
\end{array}\right) \quad B=\left(\begin{array}{ccccc}
1.6 & 1 & 0 & 0 & 0 \\
1 & 1.6 & 0 & 0 & 0 \\
0 & 0 & 1.5 & 1 & 0 \\
0 & 0 & 1 & 1.5 & 0 \\
0 & 0 & 0 & 0 & 2
\end{array}\right) \quad C=\left(\begin{array}{c}
1 \\
-2 \\
-1 \\
2 \\
-1
\end{array}\right)
$$

while Lipschitz constants $c_{1}=c_{2}=\frac{1}{2}\|A-B\|$ (see [29]). The feasible set $\mathcal{K} \subset \mathbb{R}^{5}$ is

$$
\mathcal{K}:=\left\{x \in \mathbb{R}^{5}:-5 \leq x_{i} \leq 5\right\} .
$$

Figures 5 and 6 and Table 2 show numerical results by letting different initial values.

Example 6.3. Suppose that $\mathcal{E}=L^{2}([0,1])$ is a Hilbert space with

$$
\|x\|=\sqrt{\int_{0}^{1}|x(t)|^{2} \mathrm{~d} t}
$$

and the inner product $\langle x, y\rangle=\int_{0}^{1} x(t) y(t) \mathrm{d} t, \forall x, y \in \mathcal{E}$. Assume that $\mathcal{K}:=\left\{x \in L^{2}([0,1]):\|x\| \leq 1\right\}$ be the unit ball. Let us define an operator $G: \mathcal{K} \rightarrow \mathcal{E}$ by

$$
G(x)(t)=\int_{0}^{1}(x(t)-H(t, s) f(x(s))) \mathrm{d} s+g(t),
$$



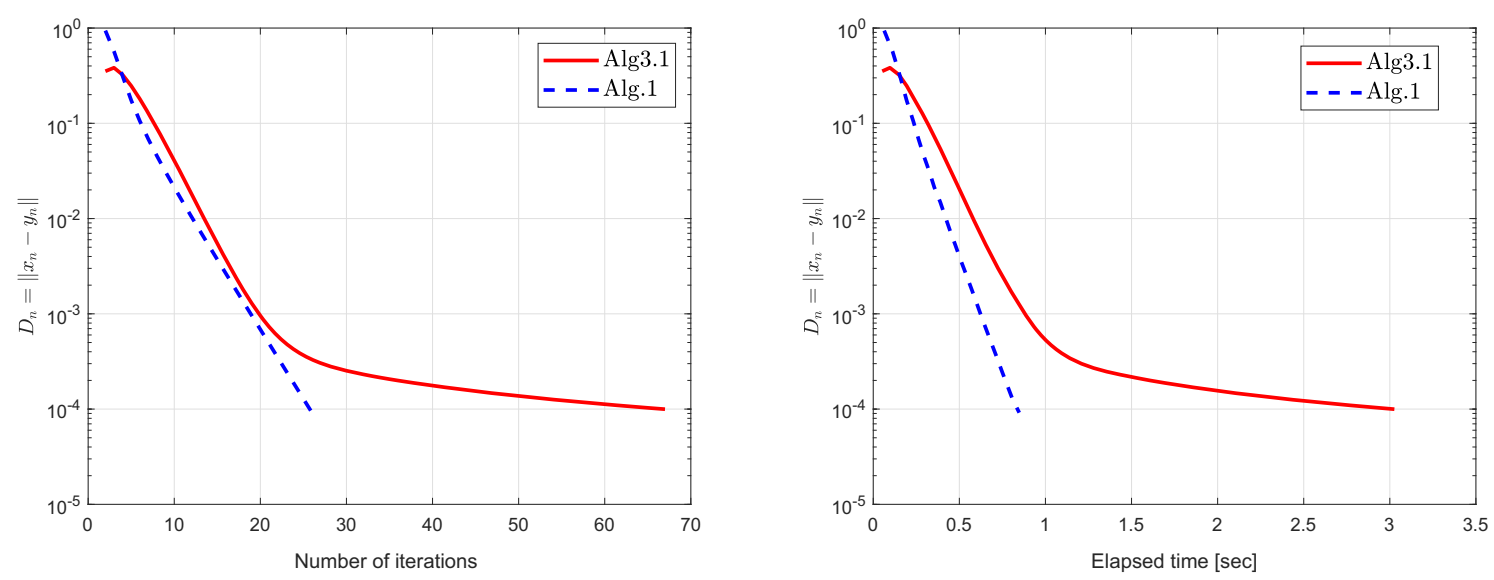

Figure 5: Example 6.2: Comparison of Algorithm 1 with Algorithm 3.1 in [31]. Comparison by using $x_{1}=(1,1,1,1,1)^{T}$.
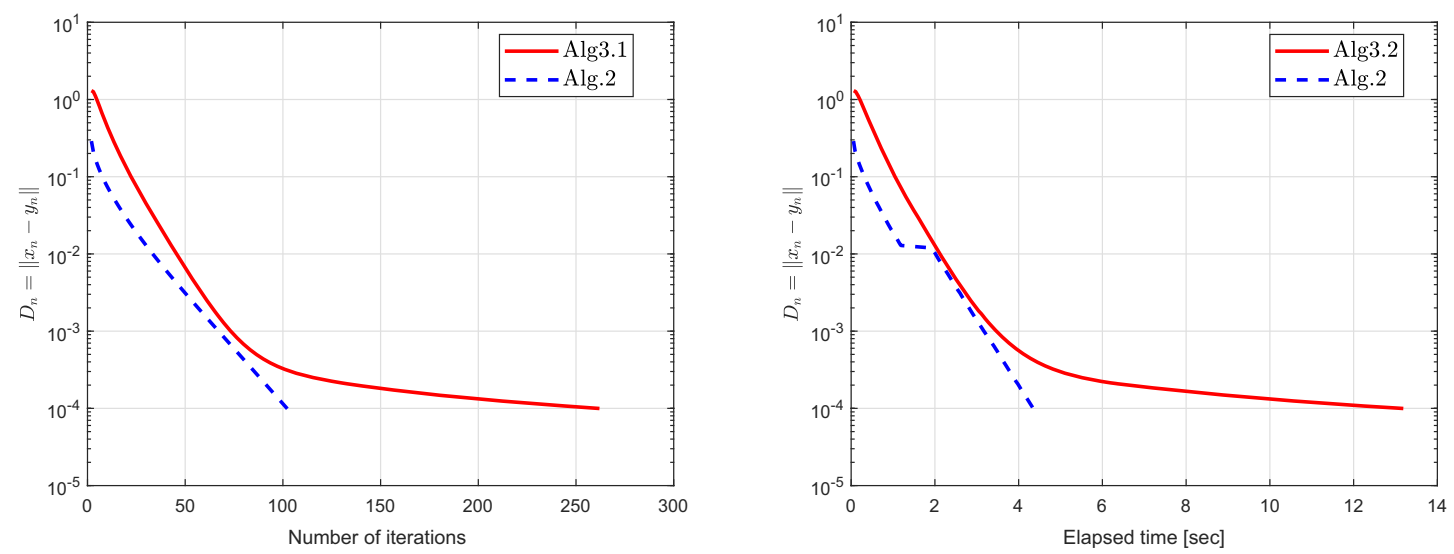

Figure 6: Example 6.2: Algorithm 2 and Algorithm 3.2 in [31]. Comparison by using $x_{1}=(1,1,1,1,1)^{T}$.

Table 2: Example 6.2: Algorithmic comparison of Algorithms 1-2 with Algorithms 3.1-3.2 in [31]

\begin{tabular}{|c|c|c|c|c|c|c|c|c|}
\hline \multirow[t]{2}{*}{$x_{1}$} & \multicolumn{4}{|c|}{ Number of iteration } & \multicolumn{4}{|c|}{ CPU time in seconds } \\
\hline & Alg3.1 & Alg.1 & Alg3.2 & Alg.2 & Alg3.1 & Alg.1 & Alg3.2 & Alg. 2 \\
\hline$(0,0,0,0,0)$ & 67 & 28 & 260 & 108 & 3.0321 & 0.8480 & 13.1667 & 4.5632 \\
\hline$(1,1,1,1,1)$ & 67 & 26 & 262 & 103 & 3.0266 & 3.8970 & 13.1867 & 4.3772 \\
\hline$(2,-1,-1,2,2)$ & 79 & 32 & 261 & 103 & 3.2310 & 1.0240 & 13.1867 & 4.3512 \\
\hline$(1,-2,3,-4,5)$ & 69 & 30 & 262 & 101 & 3.4487 & 1.0021 & 13.1867 & 4.3123 \\
\hline$(2,-1,3,-4,5)$ & 75 & 37 & 264 & 108 & 3.8215 & 1.5810 & 13.1867 & 4.4987 \\
\hline
\end{tabular}

where

$$
H(t, s)=\frac{2 t s e^{(t+s)}}{e \sqrt{e^{2}-1}}, \quad f(x)=\cos x, \quad g(t)=\frac{2 t e^{t}}{e \sqrt{e^{2}-1}}
$$


Table 3: Example 6.3: Algorithmic comparison of Algorithms 1-2 with Algorithms 3.1-3.2 in [31]

\begin{tabular}{|c|c|c|c|c|c|c|c|c|}
\hline \multirow[t]{2}{*}{$x_{1}$} & \multicolumn{4}{|c|}{ Number of iteration } & \multicolumn{4}{|c|}{ CPU time in seconds } \\
\hline & Alg3.1 & Alg.1 & Alg3.2 & Alg.2 & Alg3.1 & Alg.1 & Alg3.2 & Alg. 2 \\
\hline$t$ & 33 & 5 & 83 & 11 & 0.005131 & 0.0007435 & 0.01423 & 0.001465 \\
\hline $2 t^{2}$ & 36 & 6 & 84 & 15 & 0.005243 & 0.0007876 & 0.01612 & 0.001786 \\
\hline$e^{t}$ & 45 & 9 & 97 & 22 & 0.006754 & 0.0009867 & 0.01922 & 0.001987 \\
\hline $\sin (t)$ & 40 & 6 & 84 & 19 & 0.004987 & 0.0007927 & 0.01677 & 0.001699 \\
\hline
\end{tabular}

As shown in [41], $G$ is monotone and $L$-Lipschitz-continuous through $L=2$. Table 3 shows the numerical results by taking different initial values.

\section{Conclusion}

We have provided two extragradient-like methods to figure out a pseudo-monotone EP that requires the Lipschitz-like condition. The algorithms use a new step-size rule that is revised on each iteration, depending on prior iterations. Strong convergence results are obtained by letting certain mild conditions on the bi-function. Many numerical experiments are presented to demonstrate the numerical behavior of the proposed methods.

Acknowledgement: Nuttapol Pakkaranang would like to thank Department of Mathematics, Phetchabun Rajabhat University.

Funding information: This research project was supported by Phetchabun Rajabhat University.

Conflict of interest: Authors state no conflict of interest.

\section{References}

[1] E. Blum and W. Oettli, From optimization and variational inequalities to equilibrium problems, Math. Student 63 (1994), 123-145.

[2] K. Fan, A minimax inequality and applications, in: O. Shisha (ed.), Inequalities III, Academic Press, New York, 1972.

[3] M. Bianchi and S. Schaible, Generalized monotone bifunctions and equilibrium problems, J. Optim. Theory Appl. 90 (1996), no. 1, 31-43.

[4] G. Mastroeni, On auxiliary principle for equilibrium problems, in: P. Daniele, F. Giannessi, A. Maugeri (eds), Equilibrium Problems and Variational Models, Nonconvex Optimization and Its Applications, vol. 68, Springer, Boston, MA, 2003, pp. 289-298.

[5] G. Bigi, M. Castellani, M. Pappalardo, and M. Passacantando, Existence and solution methods for equilibria, European J. Oper. Res. 227 (2013), 1-11.

[6] L. Muu and W. Oettli, Convergence of an adaptive penalty scheme for finding constrained equilibria, Nonlinear Anal. 18 (1992), no. 12, 1159-1166.

[7] D. Van Hieu, P. K. Quy, and L. Van Vy, Explicit iterative algorithms for solving equilibrium problems, Calcolo 56 (2019), 11.

[8] D. Van Hieu, New inertial algorithm for a class of equilibrium problems, Numer. Algorithms 80 (2019), no. 4, $1413-1436$.

[9] P. K. Anh and T. N. Hai, Novel self-adaptive algorithms for non-Lipschitz equilibrium problems with applications, J. Global Optim. 73 (2019), no. 3, 637-657. 
[10] P. N. Anh, T. T. H. Anh, and N. D. Hien, Modified basic projection methods for a class of equilibrium problems, Numer. Algorithms 79 (2018), no. 1, 139-152.

[11] H. ur Rehman, P. Kumam, Y. J. Cho, and P. Yordsorn, Weak convergence of explicit extragradient algorithms for solving equilibrium problems, J. Inequal. Appl. 2019 (2019), 282.

[12] H. ur Rehman, P. Kumam, A. B. Abubakar, and Y. J. Cho, The extragradient algorithm with inertial effects extended to equilibrium problems, Comp. Appl. Math. 39 (2020), 100.

[13] H. ur Rehman, P. Kumam, W. Kumam, M. Shutaywi, and W. Jirakitpuwapat, The inertial sub-gradient extra-gradient method for a class of pseudo-monotone equilibrium problems, Symmetry 12 (2020), no. 3, 463.

[14] P. Yordsorn, P. Kumam, H. ur Rehman, and A. H. Ibrahim, A weak convergence self-adaptive method for solving pseudomonotone equilibrium problems in a real Hilbert space, Mathematics 8 (2020), 1165.

[15] P. Yordsorn, P. Kumam, and H. ur Rehman, Modified two-step extragradient method for solving the pseudomonotone equilibrium programming in a real Hilbert space, Carpathian J. Math. 36 (2020), no. 2, 313-330.

[16] H. ur Rehman, P. Kumam, I. K. Argyros, W. Deebani, and W. Kumam, Inertial extra-gradient method for solving a family of strongly pseudomonotone equilibrium problems in real Hilbert spaces with application in variational inequality problem, Symmetry 12 (2020), 503.

[17] H. ur Rehman, P. Kumam, I. K. Argyros, N. A. Alreshidi, W. Kumam, and W. Jirakitpuwapat, A self-adaptive extra-gradient methods for a family of pseudomonotone equilibrium programming with application in different classes of variational inequality problems, Symmetry 12 (2020), 523.

[18] H. ur Rehman, P. Kumam, I. K. Argyros, M. Shutaywi, and Z. Shah, Optimization based methods for solving the equilibrium problems with applications in variational inequality problems and solution of Nash equilibrium models, Mathematics 8 (2020), 822.

[19] H. ur Rehman, P. Kumam, M. Shutaywi, N. A. Alreshidi, and W. Kumam, Inertial optimization based two-step methods for solving equilibrium problems with applications in variational inequality problems and growth control equilibrium models, Energies 13 (2020), 3292.

[20] J. K. Kim, A. Hussain, and S. Salahuddin, Existence theorems for the generalized relaxed pseudomonotone variational inequalities, Nonlinear Funct. Anal. Appl. 25 (2020), no. 1, 25-34.

[21] P. N. Anh, H. T. C. Thach, and J. K. Kim, Proximal-like subgradient methods for solving multi-valued variational inequalities, Nonlinear Funct. Anal. Appl. 25 (2020), no. 3, 437-451.

[22] J. K. Kim, Convergence theorems of iterative sequences for generalized equilibrium problems involving strictly pseudocontractive mappings in Hilbert spaces, J. Comput. Anal. Appl. 18 (2015), no. 3, 454-471.

[23] J. K. Kim and P. Majee, Modified Krasnoselski-Mann iterative method for hierarchical fixed point problem and split mixed equilibrium problem, J. Inequal. Appl. 2020 (2020), 227.

[24] P. G. Hung and L. D. Muu, The Tikhonov regularization extended to equilibrium problems involving pseudomonotone bifunctions, Nonlinear Anal. 74 (2011), no. 17, 6121-6129.

[25] I. V. Konnov, Application of the proximal point method to nonmonotone equilibrium problems, J. Optim. Theory Appl. 119 (2003), 317-333.

[26] A. Moudafi, Proximal point algorithm extended to equilibrium problems, J. Nat. Geom. 15 (1999), 91-100.

[27] P. R. Oliveira, P. S. M. Santos, and A. N. Silva, A Tikhonov-type regularization for equilibrium problems in Hilbert spaces, J. Math. Anal. Appl. 401 (2013), 336-342.

[28] S. D. Flåm and A. S. Antipin, Equilibrium programming using proximal-like algorithms, Math. Programm. 78 (1996), 29-41.

[29] D. Q. Tran, M. L. Dung, and V. H. Nguyen, Extragradient algorithms extended to equilibrium problems, Optimization 57 (2008), no. 6, 749-776.

[30] G. M. Korpelevich, The extragradient method for finding saddle points and other problems, Matecon 12 (1976), 747-756.

[31] S. Wang, Y. Zhang, P. Ping, Y. Cho, and H. Guo, New extragradient methods with non-convex combination for pseudomonotone equilibrium problems with applications in Hilbert spaces, Filomat 33 (2019), no. 6, 1677-1693.

[32] Y. Censor, A. Gibali and S. Reich, The subgradient extragradient method for solving variational inequalities in Hilbert spaces, J. Optim. Theory Appl. 148 (2014), no. 2, 318-335.

[33] J. V. Tiel, Convex Analysis: An Introductory Text, Wiley, New York, 1984.

[34] P. J. Bushell, Uniform convexity, hyperbolic geometry, and nonexpansive mappings, in: Monographs and Textbooks in Pure and Applied Mathematics, vol. 83, Marcel Dekker, Inc., New York, 1984.

[35] E. Kreyszig, Introductory Functional Analysis with Applications, John Wiley \& Sons, Inc., New York, 1989.

[36] H. H. Bauschke and P. L. Combettes, Convex Analysis and Monotone Operator Theory in Hilbert Spaces, Springer, New York, 2011.

[37] H. K. Xu, Another control condition in an iterative method for nonexpansive mappings, Bull. Austral. Math. Soc. 65 (2002), no. 1, 109-113.

[38] P. E. Maingé, Strong convergence of projected subgradient methods for nonsmooth and nonstrictly convex minimization, Set-Valued Anal. 16 (2008), no. 7-8, 899-912.

[39] F. E. Browder and W. V. Petryshyn, Construction of fixed points of nonlinear mappings in Hilbert space, J. Math. Anal. Appl. 20 (1967), 197-228. 
[40] H. ur Rehman, P. Kumam, Y. J. Cho, Y. I. Suleiman, and W. Kumam, Modified Popov's explicit iterative algorithms for solving pseudomonotone equilibrium problems, Optim. Methods Softw. 36 (2021), no. 1, 82-113.

[41] D. V. Hieu, P. K. Anh, and L. D. Muu, Modified hybrid projection methods for finding common solutions to variational inequality problems, Comput. Optim. Appl. 66 (2017), no. 1, 75-96. 